\title{
Model-based scaling of the streamwise energy density in high-Reynolds-number turbulent channels
}

\author{
Rashad Moarref $^{1}{ }^{\dagger}$, Ati S. Sharma ${ }^{2}$, Joel A. Tropp ${ }^{3}$ and Beverley J. McKeon ${ }^{1}$ \\ ${ }^{1}$ Graduate Aerospace Laboratories, California Institute of Technology, CA 91125, USA \\ ${ }^{2}$ Engineering and the Environment, University of Southampton, Southampton SO17 1BJ, UK \\ ${ }^{3}$ Computing \& Mathematical Sciences, California Institute of Technology, CA 91125, USA
}

(Received 8 February 2013; revised 20 August 2013; accepted 24 August 2013; first published online 9 October 2013)

We study the Reynolds-number scaling and the geometric self-similarity of a gainbased, low-rank approximation to turbulent channel flows, determined by the resolvent formulation of McKeon \& Sharma (J. Fluid Mech., vol. 658, 2010, pp. 336-382), in order to obtain a description of the streamwise turbulence intensity from direct consideration of the Navier-Stokes equations. Under this formulation, the velocity field is decomposed into propagating waves (with single streamwise and spanwise wavelengths and wave speed) whose wall-normal shapes are determined from the principal singular function of the corresponding resolvent operator. Using the accepted scalings of the mean velocity in wall-bounded turbulent flows, we establish that the resolvent operator admits three classes of wave parameters that induce universal behaviour with Reynolds number in the low-rank model, and which are consistent with scalings proposed throughout the wall turbulence literature. In addition, it is shown that a necessary condition for geometrically self-similar resolvent modes is the presence of a logarithmic turbulent mean velocity. Under the practical assumption that the mean velocity consists of a logarithmic region, we identify the scalings that constitute hierarchies of self-similar modes that are parameterized by the critical wall-normal location where the speed of the mode equals the local turbulent mean velocity. For the rank-1 model subject to broadband forcing, the integrated streamwise energy density takes a universal form which is consistent with the dominant near-wall turbulent motions. When the shape of the forcing is optimized to enforce matching with results from direct numerical simulations at low turbulent Reynolds numbers, further similarity appears. Representation of these weight functions using similarity laws enables prediction of the Reynolds number and wall-normal variations of the streamwise energy intensity at high Reynolds numbers $\left(R e_{\tau} \approx 10^{3}-10^{10}\right)$. Results from this low-rank model of the Navier-Stokes equations compare favourably with experimental results in the literature.

Key words: mathematical foundations, Navier-Stokes equations, turbulent boundary layers 


\section{Introduction}

Understanding the behaviour of wall-bounded turbulent flows at high Reynolds numbers has tremendous technological implications, for example, in air and water transportation. This problem has received significant attention over the last two decades especially in the light of full-field flow information revealed by direct numerical simulations (DNS) at relatively small Reynolds numbers and high-Reynoldsnumber experiments. Notwithstanding the recent developments, the highest Reynolds numbers that are considered in DNS are an order of magnitude smaller than experiments, which are in turn conducted at Reynolds numbers that are typically two orders of magnitude smaller than most applications. This creates a critical demand for model-based approaches that describe and predict the behaviour of turbulent flows at technologically relevant Reynolds numbers.

Wall turbulence has been the topic of several reviews; see, for example, Robinson (1991) and Adrian (2007) for structure of coherent motions, Gad-El-Hak \& Bandyopadhyay (1994) for turbulence statistics and scaling issues, Panton (2001) for self-sustaining turbulence mechanisms, and Klewicki (2010), Marusic et al. (2010c) and Smits, McKeon \& Marusic (2011) for the latest findings and main challenges in examining high-Reynolds-number wall turbulence. In the present study, special attention is paid to scaling, universality, and geometric self-similarity of the turbulent energy spectra at high Reynolds numbers. We also note that the energy spectra exhibit clear signatures of coherent turbulent motions such as the near-wall streaks, the largescale motions (LSMs), and the very large-scale motions (VLSMs).

\subsection{Overview of dominant coherent motions}

In the interests of giving a brief overview of the energetically dominant coherent motions in wall turbulence, we will review three classes of structure. The near-wall system of quasi-streamwise streaks and counter-rotating vortices with streamwise length and spanwise spacing of approximately 1000 and 100 inner (viscous) units, centred at approximately 15 inner units above the wall, has been well-studied. These ubiquitous features of wall turbulence are responsible for large production of turbulent kinetic energy (Kline et al. 1967; Smith \& Metzler 1983).

Another commonly observed feature of turbulent flows is the hairpin vortex. In low-Reynolds-number flows, at least, packets of hairpin vortices have been observed to extend from the wall to the edge of the boundary layer and constitute LSMs (Head \& Bandyopadhyay 1981; Adrian, Meinhart \& Tomkins 2000; Adrian 2007), with streamwise extent approximately $2-3$ outer units (channel half-height, pipe radius, or the boundary layer thickness).

VLSMs have been observed to reside in the logarithmic region of the turbulent mean velocity, with lengths of approximately 10-15 outer units in boundary layers and up to 30 outer units in channels and pipes (see, for example, Kim \& Adrian 1999; Balakumar \& Adrian 2007; Monty et al. 2007). The emergence of VLSMs was originally attributed to alignment of LSMs (Kim \& Adrian 1999). However, Smits et al. (2011) concluded that this is unlikely since the detached LSMs are located at a farther distance from the wall than the VLSMs and the attached LSMs have much smaller width than VLSMs and are convected at different speeds. Recently, the correlation between the envelope of small-scale activity and the large-scale velocity signal (identified via filtering in spectral space), which has been interpreted as an amplitude modulation of the small scales, has been investigated in detail, see e.g. Hutchins \& Marusic (2007b), Mathis, Hutchins \& Marusic (2009a), Mathis et al. (2009b), Chung \& McKeon (2010) and Hutchins et al. (2011). 


\subsection{Overview of scaling issues}

In spite of recent advances in understanding the structure of wall turbulence, the Reynolds-number scaling of the turbulent energy spectra and the energy intensities remains an open area of research. The main experimental obstacle is maintaining the necessary spatial resolution for measurement accuracy while achieving the high Reynolds numbers required for large separation between the small and large turbulent scales. For example, the available experiments are performed at relatively small friction Reynolds numbers, $R e_{\tau} \approx O\left(10^{4}\right)$, with a notable exception of the atmospheric surface layer measurements of e.g. Metzger \& Klewicki $(2001)\left(R e_{\tau} \approx O\left(10^{6}\right)\right)$ that are in turn generally contaminated by surface roughness effects. Most high-Reynoldsnumber experiments suffer from spatial resolution issues in the inner region (see, for example, Hutchins et al. 2009).

Significant experimental effort has been devoted to determining the behaviour of the streamwise energy intensity at high Reynolds numbers since it dominates the turbulent kinetic energy and is easier to measure relative to the wall-normal and spanwise velocities. It is understood that both small and large scales contribute to the streamwise energy intensity (Metzger \& Klewicki 2001; Marusic \& Kunkel 2003; Hutchins \& Marusic 2007a; Marusic, Mathis \& Hutchins 2010a). It is wellknown that a region of the streamwise wavenumber spectrum scales with inner units; Marusic et al. (2010a) showed by filtering that the contribution of such scales to the streamwise energy intensity, and therefore by extension also the streamwise spectrum, is universal, i.e. independent of Reynolds number. On the other hand, the large motions have been shown to scale in outer units (Kim \& Adrian 1999); Mathis et al. (2009a) proposed that the corresponding peak in streamwise intensity occurs close to the geometric mean of the limits of the logarithmic region in the turbulent mean velocity. The amplitude of this energetic peak increases with Reynolds number and has a footprint down to the wall (Hutchins \& Marusic 2007b). Using data from experiments of canonical wall-bounded turbulent flows, Alfredsson, Örlü \& Segalini (2012) proposed a composite profile for the streamwise turbulence intensity and showed the possibility of an outer peak at high Reynolds numbers. Note however, that available data are not sufficiently well-resolved to determine unequivocally the Reynolds-number scaling of either the inner or outer peaks of the streamwise energy intensity (see, for example, Marusic et al. 2010a).

Theoretical approaches also offer insight into the scaling of the spectrum with increasing Reynolds numbers, originating with the attached-eddy concepts described by Townsend (1976). These eddies are attached in the sense that their height scales with their distance from the wall, and they are geometrically self-similar since their wall-parallel length scales are proportional to their height. Perry \& Chong (1982) developed these ideas to include hierarchies of geometrically self-similar attached eddies in the logarithmic region of the turbulent mean velocity. They systematically predicted that if the population density of the attached eddies inversely decreases with their height, both the turbulent mean velocity and the wall-parallel energy intensities exhibit logarithmic dependence on the distance from the wall. The logarithmic behaviour of the mean velocity and the streamwise energy intensity was recently confirmed using high-Reynolds-number experiments (Marusic et al. 2013). However, the attached-eddy hypothesis does not predict the exact shape of the eddies or their evolution in time.

Subsequent works by Perry and co-authors extended the attached-eddy formulation beyond the logarithmic region; Marusic \& Kunkel (2003) used empirical scaling arguments concerning the effective forcing of the outer turbulence on the viscous 
region to propose a similarity expression for the streamwise energy intensity that is valid throughout the zero-pressure boundary layer. Recently, Marusic, Mathis \& Hutchins $(2010 b)$ outlined an observationally based, predictive formulation for the variation of the streamwise turbulent intensity up to the geometric mean of the logarithmic region based on consideration of the correlation between large and small scales. Most recently, Mizuno \& Jiménez (2013) used DNS to show that self-similarity of the velocity fluctuations is sufficient and seemingly important for reproducing a logarithmic profile in the mean velocity. They also observed that the logarithmic region can be maintained independent of the near-wall dynamics.

\subsection{Review of previous model-based approaches}

We seek in this work a description of the streamwise turbulence intensity for all wall-normal locations arising from direct consideration, and modelling, of the Navier-Stokes equations (NSE). There has been much work in this vein, highlighting several important features of the NSE. We provide a brief review of the most relevant literature here.

The critical role of linear amplification mechanism in promoting and maintaining turbulent flows was highlighted in DNS of Kim \& Lim (2000). In addition, it was shown that nonlinearity plays an important role in regenerating the near-wall region of turbulent shear flows through a self-sustaining process (Hamilton, Kim \& Waleffe 1995; Waleffe 1997; Schoppa \& Hussain 2002). More recently, significant effort has been directed at identification and analysis of exact solutions of the NSE, such as travelling waves and periodic orbits, see e.g. Waleffe (2003) and Wedin \& Kerswell (2004) and the review paper by Kerswell (2005).

It is understood that high sensitivity of the laminar flow to disturbances provides alternative paths to transition that bypass linear instability; see, for example, Schmid \& Henningson (2001). Trefethen et al. (1993) showed that the high flow sensitivity is related to non-normality of the coupled Orr-Sommerfeld and Squire operators; see also Schmid (2007). These operators are coupled in the presence of mean shear and spanwise-varying fluctuations. Physically, as originally explained by Landahl (1975), a large streamwise disturbance is induced on the flow in response to lift-up of a fluid particle by the wall-normal velocity such that its wall-parallel momentum is conserved.

Even in linearly stable flows, the high sensitivity can result in large transient responses, meaning that the energy of certain initial perturbations significantly grows before eventual decay to zero (Gustavsson 1991; Butler \& Farrell 1992; Klingmann 1992; Reddy \& Henningson 1993; Schmid \& Henningson 1994). In addition, the high sensitivity is responsible for high energy amplification, meaning that the velocity fluctuations achieve a large variance at the steady state for the flow subject to zero-mean stochastic disturbances (Farrell \& Ioannou 1993b; Bamieh \& Dahleh 2001; Jovanović \& Bamieh 2005). The dominant structures that emerge from the above transient growth and energy amplification analyses are reminiscent of the streamwise streaks observed at the early stages of transition to turbulence (Matsubara \& Alfredsson 2001). They are characterized by infinitely long spanwise-periodic regions of high and low streamwise velocity associated with pairs of counter-rotating streamwise vortices that are separated by approximately 3.5 outer units.

It is believed that the NSE linearized around the turbulent mean velocity are stable for all Reynolds numbers (Malkus 1956; Reynolds \& Tiederman 1967). Early modelbased approaches extended the aforementioned sensitivity analyses of the laminar flow to the turbulent channel flow and found dominance of streamwise streaks that are spaced by 3 outer units, which is approximately the same as in the laminar flow. In 
addition to the outer-scaled dominant structures, Butler \& Farrell (1993) and Farrell \& Ioannou (1993a) showed that the largest transient response over an eddy turnover time of 80 inner units, associated with the near-wall cycle, is obtained for initial perturbations that are infinitely long and have the same spanwise spacing as the near-wall streaks, i.e. 100 inner units. The same streamwise and spanwise lengths were obtained in flows subject to stochastic disturbances over a coherence time of 90 inner units (Farrell \& Ioannou 1998).

Reynolds \& Hussain (1972) put forward a modified linear model to account for the effect of background Reynolds stresses on the velocity fluctuations. They proposed to augment the molecular viscosity by the turbulent eddy viscosity that is required to maintain the mean velocity. This model yields two local optima for the structures with largest transient growth (del Álamo \& Jiménez 2006; Pujals et al. 2009) and energy amplification (Hwang \& Cossu 2010) without the need for confining the optimization time. These peaks correspond to streamwise-elongated structures with a spacing of 80 inner units and 3-4 outer units and are in fair agreement with the spacing of nearwall streaks and the very large-scale motions in real turbulent flows. The geometric similarity of the optimal transient response to initial perturbations and the optimal responses to harmonic and stochastic forcings was highlighted by Hwang \& Cossu (2010) using the linearized NSE with turbulent eddy viscosity. These authors found that the streamwise-constant optimal responses scale with the spanwise wavelength in the wall-normal direction for spanwise wavelengths between the inner- and outerscaled regions.

An exact representation of the NSE was introduced by McKeon \& Sharma (2010) in which: (i) a set of linear sub-systems describe extraction of energy from the mean velocity at individual wavenumbers/frequencies; and (ii) the only source of coupling between these sub-systems is the conservative nonlinear interaction of their outputs, that determines both the input to the sub-systems and the turbulent mean velocity. At its heart is the ability to analyse the flow of energy from the mean velocity to all the velocity scales and identify the essential linear amplification and nonlinear redistribution mechanisms that drive the turbulent flow. The input-output relationship of the linear sub-systems can be described by transfer functions whose low-rank nature in the wall-normal direction enables significant simplification of their analysis.

One of the main differences between the formulation of McKeon \& Sharma (2010) and other input-output analyses of laminar and turbulent flows (see, for example, Jovanović \& Bamieh 2005; Hwang \& Cossu 2010) is parameterization of the waves with wave speed rather than temporal frequency. The latter approaches showed that the globally optimal transient growth and energy amplification takes place for zero streamwise wavenumber and temporal frequency. Selecting the wave speed, as emphasized by McKeon \& Sharma (2010): (i) enables a systematic search for both locally (in wall-normal direction) and globally optimal wave shapes and parameters; (ii) removes the ambiguity about the wave speed corresponding to the globally optimal waves by determining the limit of the ratio between zero streamwise wavenumber and temporal frequency; and (iii) distinguishes between non-normality and critical behaviour as the main linear amplification mechanisms.

McKeon \& Sharma (2010) showed that the principal forcing and response directions associated with the linear sub-systems are consistent with the dominant response shapes in real turbulent pipe flows. In addition, the low-dimensional and sparse feature of the resulting model enables development and utilization of compressive sampling techniques for analysing the turbulent flow dynamics (Bourguignon et al. 2013). This formulation has also proven useful for pre- and post-diction of experimental 
observations in turbulent pipe flow (McKeon, Sharma \& Jacobi 2013; Sharma \& McKeon 2013).

\subsection{Paper outline}

In this paper, we identify the Reynolds-number scaling of a low-rank approximation to turbulent channel flow and utilize it for predicting the streamwise energy intensity at high Reynolds numbers. Our development is outlined as follows. In $\S 2$, we briefly review the resolvent formulation, highlight its low-rank nature, and show that a rank-1 approximation captures the characteristics of the most energetic modes of real turbulent channels. The stage is set for studying the energy density of fluctuations using a minimum number of assumptions by considering a rank-1 model in the wallnormal direction subject to broadband forcing in the wall-parallel directions and time. Furthermore, a summary of the computational approach for determining the rank-1 model is provided.

Three classes of wave parameters for which the low-rank approximation of the resolvent exhibits universal behaviour (independence) with Reynolds number are identified in $\S 3$. The requirement for universality highlights the role of wave speed in distinguishing these classes. Each class of waves is characterized by a unique range of wave speeds and a unique spatial scaling that emerge from the resolvent. For the rank-1 model subject to broadband forcing, we reveal the universal streamwise energy densities, and show that the peaks of these energy densities roughly agree with the most energetic turbulent motions, i.e. the near-wall streaks, the VLSMs, and the LSMs.

In $\S 4$, we show that the streamwise energy density of the rank-1 model with broadband forcing can be optimally weighted as a function of wave speed to match the intensity of simulations at low turbulent Reynolds numbers. The weight functions are then formulated using similarity laws which, in conjunction with the universal energy densities, enable prediction of the streamwise energy intensity at high Reynolds numbers. The paper is concluded in $\S 5$ and limitations and several future directions are discussed.

\section{Low-rank approximation to channel flow}

An overview of the rationale for considering a low-rank approximation to turbulent channel flow is presented in this section. We follow the development of McKeon \& Sharma (2010) for turbulent pipe flow, showing that equivalent results are obtained for channels and highlighting the new observations.

The pressure-driven flow of an incompressible Newtonian fluid is governed by the non-dimensional NSE and the continuity constraint

$$
\left.\begin{array}{l}
\boldsymbol{u}_{t}+(\boldsymbol{u} \cdot \nabla) \boldsymbol{u}+\nabla P=\left(1 / R e_{\tau}\right) \Delta \boldsymbol{u}, \\
\nabla \cdot \boldsymbol{u}=0,
\end{array}\right\}
$$

where $\boldsymbol{u}(x, y, z, t)$ is the velocity vector, $P(x, y, z, t)$ is the pressure, $\boldsymbol{\nabla}$ is the gradient operator, and $\Delta=\nabla \cdot \nabla$ is the Laplacian. The streamwise and spanwise directions, $x$ and $z$, are infinitely long, the wall-normal direction is finite, $0 \leqslant y \leqslant 2$, and $t$ denotes time; see figure $1(a)$ for the geometry. The subscript $t$ represents temporal derivative, e.g. $\boldsymbol{u}_{t}=\partial \boldsymbol{u} / \partial t$. The Reynolds number $\operatorname{Re}_{\tau}=u_{\tau} h / v$ is defined based on the channel half-height $h$, kinematic viscosity $\nu$, and friction velocity $u_{\tau}=\sqrt{\tau_{w} / \rho}$, where $\tau_{w}$ is the shear stress at the wall, and $\rho$ is the density. Velocity is normalized by $u_{\tau}$, spatial variables by $h$, time by $h / u_{\tau}$, and pressure by $\rho u_{\tau}^{2}$. The spatial variables are denoted by ${ }^{+}$when normalized by the viscous length scale $v / u_{\tau}$, e.g. $y^{+}=R e_{\tau} y$. 
(a)

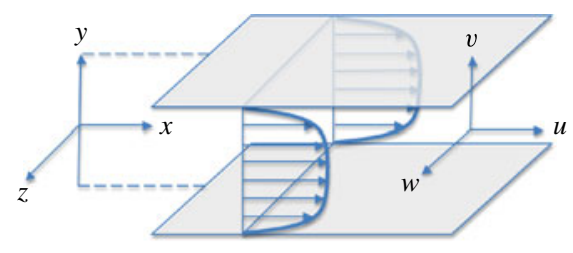

(b)

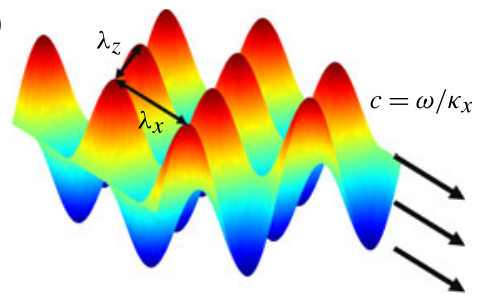

FIgURE 1. (Colour online) (a) Pressure-driven channel flow. (b) Schematic of a twodimensional propagating wave with streamwise and spanwise wavelengths $\lambda_{x}$ and $\lambda_{z}$ and streamwise speed $c$.

\subsection{Decomposition in homogeneous directions}

The velocity is decomposed using the Fourier transform in the homogeneous directions and time

$$
\boldsymbol{u}(x, y, z, t)=\iiint_{-\infty}^{\infty} \hat{\boldsymbol{u}}\left(y ; \kappa_{x}, \kappa_{z}, \omega\right) \mathrm{e}^{\mathrm{i}\left(\kappa_{x} x+\kappa_{z} z-\omega t\right)} \mathrm{d} \kappa_{x} \mathrm{~d} \kappa_{z} \mathrm{~d} \omega,
$$

where the hat denotes a variable in the transformed domain, and the triplet $\left(\kappa_{x}, \kappa_{z}, \omega\right)$ is the streamwise and spanwise wavenumbers and the temporal (angular) frequency. The Fourier basis is optimal in the homogeneous wall-parallel directions. It is also an appropriate basis in time under stationary conditions. For any $\left(\kappa_{x}, \kappa_{z}, \omega\right) \neq$ $0, \hat{\boldsymbol{u}}\left(y ; \kappa_{x}, \kappa_{z}, \omega\right)$ represents a propagating wave with streamwise and spanwise wavelengths $\lambda_{x}=2 \pi / \kappa_{x}$ and $\lambda_{z}=2 \pi / \kappa_{z}$ and speed $c=\omega / \kappa_{x}$ in the streamwise direction; see figure $1(b)$ for an illustration. Some special cases include standing waves $(c=0)$, infinitely long waves $\left(\kappa_{x}=0\right)$, and infinitely wide waves $\left(\kappa_{z}=0\right)$. In this study, we emphasize the eminent role of wave speed, a factor that was highlighted by McKeon \& Sharma (2010) while being predominantly neglected in the previous studies, in determining the classes of propagating waves that are universal with Reynolds number.

The turbulent mean velocity $\boldsymbol{U}(y)=\left[\begin{array}{lll}U(y) & 0 & 0\end{array}\right]^{\mathrm{T}}=\hat{\boldsymbol{u}}(y ; 0,0,0)$ corresponds to $\left(\kappa_{x}, \kappa_{z}, \omega\right)=0$ and is assumed to be known. Note that our main results, i.e. the identified scalings in $\S 3$, rely on the accepted scales of the turbulent mean velocity and, otherwise, do not depend on the exact shape of $U$. McKeon \& Sharma (2010) avoided the closure problem for the mean velocity by using $U(y)$ obtained in pipe flow experiments, but note that the resolvent formulation could be used to determine the mean velocity profile, a topic of ongoing work (see McKeon et al. 2013). Here, we use a semi-empirical turbulent viscosity model, originally proposed for pipe flow (Malkus 1956; Cess 1958) and extended to channel flow (Reynolds \& Tiederman 1967), to determine $U(y)$ :

$$
\begin{aligned}
U(y) & =\operatorname{Re}_{\tau} \int_{0}^{y} \frac{1-\xi}{1+v_{T}(\xi)} \mathrm{d} \xi \\
\nu_{T}(y) & =\frac{1}{2}\left\{1+\left(\frac{\kappa e_{\tau}}{3}\left(2 y-y^{2}\right)\left(3-4 y+2 y^{2}\right)\left\{1-\mathrm{e}^{(|y-1|-1) \operatorname{Re}_{\tau} / \alpha}\right\}\right)^{2}\right\}^{1 / 2}-\frac{1}{2},
\end{aligned}
$$

where $v_{T}$ is normalized by $\nu$, and the parameters $\alpha$ and $\kappa$ appear in the van Driest wall law and the von Kármán log law. These parameters are obtained by minimizing 


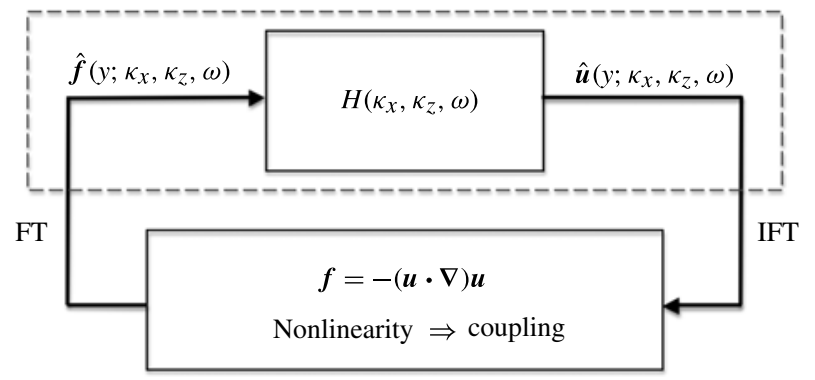

FIGURE 2. For any triplet $\left(\kappa_{x}, \kappa_{z}, \omega\right)$, the operator $H\left(\kappa_{x}, \kappa_{z}, \omega\right)$ maps the forcing $\hat{f}$ to the response $\hat{\boldsymbol{u}}$. The different wavenumbers are coupled via the quadratic relationship between $\boldsymbol{f}(x, y, z, t)$ and $\boldsymbol{u}(x, y, z, t)$. FT and IFT stand for Fourier transform and inverse Fourier transform, respectively. The input-output map (shown with the dashed rectangle) is the main focus of the present study.

the deviation between $U(y)$ in (2.3) and the DNS-based turbulent mean velocity profile. The $\alpha$ and $\kappa$ obtained for $R e_{\tau}=186,547$ and 934 (Moarref \& Jovanović 2012) suggest that both of these values converge for large $R e_{\tau}$. We take $\alpha=25.4$ and $\kappa=0.426$ for all Reynolds numbers and note that these values are optimized for $R e_{\tau}=2003$ (del Álamo \& Jiménez 2006; Pujals et al. 2009).

Following McKeon \& Sharma (2010), the convective nonlinearity in (2.1) is considered as a forcing term $\boldsymbol{f}=-(\boldsymbol{u} \cdot \nabla) \boldsymbol{u}$ that drives the velocity fluctuations, see also figure 2. For any $\left(\kappa_{x}, \kappa_{z}, \omega\right) \neq 0$, an equation for velocity fluctuations $\hat{\boldsymbol{u}}\left(y ; \kappa_{x}, \kappa_{z}, \omega\right)=[\hat{u} \hat{v} \hat{w}]^{\mathrm{T}}$ around the turbulent mean velocity is obtained by substituting (2.2) in (2.1), and using the orthonormality of the complex exponential functions:

$$
\begin{array}{r}
-\mathrm{i} \omega \hat{\boldsymbol{u}}+(\boldsymbol{U} \cdot \nabla) \hat{\boldsymbol{u}}+(\hat{\boldsymbol{u}} \cdot \nabla) \boldsymbol{U}+\nabla \hat{p}-\left(1 / \operatorname{Re}_{\tau}\right) \Delta \hat{\boldsymbol{u}}=\hat{\boldsymbol{f}}, \\
\nabla \cdot \hat{\boldsymbol{u}}=0 .
\end{array}
$$

Here, $\nabla=\left[\begin{array}{lll}\mathrm{i} \kappa_{x} & \partial_{y} & \mathrm{i} \kappa_{z}\end{array}\right]^{\mathrm{T}}, \Delta=\partial_{y y}-\kappa^{2}$ with $\kappa^{2}=\kappa_{x}^{2}+\kappa_{z}^{2}$, and

$$
\begin{aligned}
\hat{\boldsymbol{f}}\left(y ; \kappa_{x}, \kappa_{z}, \omega\right)=\left[\hat{f}_{1} \hat{f}_{2} \hat{f}_{3}\right]^{\mathrm{T}}= & \iiint_{\substack{\left(\kappa_{x}^{\prime}, \kappa_{z}^{\prime}, \omega^{\prime}\right) \neq(0,0,0) \\
\left(\kappa_{x}^{\prime}, \kappa_{2}^{\prime}, \omega^{\prime}\right) \neq\left(\kappa_{x}, \kappa_{z}, \omega\right)}}\left(\hat{\boldsymbol{u}}\left(y ; \kappa_{x}^{\prime}, \kappa_{z}^{\prime}, \omega^{\prime}\right) \cdot \nabla\right) \\
& \times \hat{\boldsymbol{u}}\left(y ; \kappa_{x}-\kappa_{x}^{\prime}, \kappa_{z}-\kappa_{z}^{\prime}, \omega-\omega^{\prime}\right) \mathrm{d} \kappa_{x}^{\prime} \mathrm{d} \kappa_{z}^{\prime} \mathrm{d} \omega^{\prime} .
\end{aligned}
$$

McKeon \& Sharma (2010) implicitly accounted for the continuity constraint by projecting the velocity field onto the divergence-free basis of Meseguer \& Trefethen (2003). Here, we use a standard choice of wall-normal velocity $\hat{v}$ and wall-normal vorticity $\hat{\eta}=\mathrm{i} \kappa_{z} \hat{u}-\mathrm{i} \kappa_{x} \hat{w}$ as the state variables, $\hat{\zeta}\left(y ; \kappa_{x}, \kappa_{z}, \omega\right)=[\hat{v} \hat{\eta}]^{\mathrm{T}}$, to eliminate the pressure term and the continuity constraint from (2.4) and obtain

$$
\begin{aligned}
-\left(\mathrm{i} \omega I+A\left(\kappa_{x}, \kappa_{z}\right)\right) \hat{\zeta}\left(y ; \kappa_{x}, \kappa_{z}, \omega\right) & =C^{\dagger}\left(\kappa_{x}, \kappa_{z}\right) \hat{\boldsymbol{f}}\left(y ; \kappa_{x}, \kappa_{z}, \omega\right), \\
\hat{\boldsymbol{u}}\left(y ; \kappa_{x}, \kappa_{z}, \omega\right) & =C\left(\kappa_{x}, \kappa_{z}\right) \hat{\boldsymbol{\zeta}}\left(y ; \kappa_{x}, \kappa_{z}, \omega\right) .
\end{aligned}
$$

Here, $A$ is the state operator, $C$ maps the state vector to the velocity vector, and the adjoint of $C$ (denoted by $C^{\dagger}$ ) maps the forcing vector to the state vector. $A, C$, and $C^{\dagger}$ 
are operators in $y$ and parameterized by $\kappa_{x}$ and $\kappa_{z}$ :

$$
\begin{aligned}
& A=\left[\begin{array}{cc}
\Delta^{-1}\left(\left(1 / R e_{\tau}\right) \Delta^{2}+\mathrm{i} \kappa_{x}\left(U^{\prime \prime}-U \Delta\right)\right) & 0 \\
-\mathrm{i} \kappa_{z} U^{\prime} & \left(1 / R e_{\tau}\right) \Delta-\mathrm{i} \kappa_{x} U
\end{array}\right], \\
& C=\frac{1}{\kappa^{2}}\left[\begin{array}{cc}
\mathrm{i} \kappa_{x} \partial_{y} & -\mathrm{i} \kappa_{z} \\
\kappa^{2} & 0 \\
\mathrm{i} \kappa_{z} \partial_{y} & \mathrm{i} \kappa_{x}
\end{array}\right], \quad C^{\dagger}=\left[\begin{array}{ccc}
-\mathrm{i} \kappa_{x} \Delta^{-1} \partial_{y} & \kappa^{2} \Delta^{-1} & -\mathrm{i} \kappa_{z} \Delta^{-1} \partial_{y} \\
\mathrm{i} \kappa_{z} & 0 & -\mathrm{i} \kappa_{x}
\end{array}\right],
\end{aligned}
$$

where $\Delta^{2}=\partial_{y y y y}-2 \kappa^{2} \partial_{y y}+\kappa^{4}$, and the prime denotes differentiation in $y$, e.g. $U^{\prime}(y)=\mathrm{d} U / \mathrm{d} y$. The input-output relationship between $\hat{\boldsymbol{f}}$ and $\hat{\boldsymbol{u}}$ is obtained upon elimination of $\hat{\zeta}$ from (2.6):

$$
\begin{array}{r}
\hat{\boldsymbol{u}}\left(y ; \kappa_{x}, \kappa_{z}, \omega\right)=H\left(\kappa_{x}, \kappa_{z}, \omega\right) \hat{\boldsymbol{f}}\left(y ; \kappa_{x}, \kappa_{z}, \omega\right), \\
H\left(\kappa_{x}, \kappa_{z}, \omega\right)=C\left(\kappa_{x}, \kappa_{z}\right) R_{A}\left(\kappa_{x}, \kappa_{z}, \omega\right) C^{\dagger}\left(\kappa_{x}, \kappa_{z}\right),
\end{array}
$$

where $R_{A}\left(\kappa_{x}, \kappa_{z}, \omega\right)=-\left(\mathrm{i} \omega I+A\left(\kappa_{x}, \kappa_{z}\right)\right)^{-1}$ is the resolvent of $A$ :

$$
R_{A}=\left[\begin{array}{cc}
\Delta^{-1}\left(\mathrm{i} \kappa_{x}\left((U-c) \Delta-U^{\prime \prime}\right)-\left(1 / R e_{\tau}\right) \Delta^{2}\right) & 0 \\
\mathrm{i} \kappa_{z} U^{\prime} & \mathrm{i} \kappa_{x}(U-c)-\left(1 / R e_{\tau}\right) \Delta
\end{array}\right]^{-1}
$$

As illustrated in figure 2, the only source of coupling between propagating waves with different wavenumbers is the quadratic dependence of $\boldsymbol{f}(x, y, z, t)$ on $\boldsymbol{u}(x, y, z, t)$. For any wavenumber triplet, the input-output map from $\hat{\boldsymbol{f}}$ to $\hat{\boldsymbol{u}}$ (shown by the dashed rectangle) represents a sub-system of the full NSE.

\subsection{Decomposition in the wall-normal direction}

The transfer function $H\left(\kappa_{x}, \kappa_{z}, \omega\right)$ provides a large amount of information about the input-output relationship between $\hat{\boldsymbol{f}}$ and $\hat{\boldsymbol{u}}$. Following the gain analysis of McKeon \& Sharma (2010), we use the Schmidt (singular value) decomposition to provide a wall-normal basis based on the most highly amplified forcing and response directions:

$$
\begin{aligned}
\hat{\boldsymbol{u}}\left(y ; \kappa_{x}, \kappa_{z}, \omega\right) & =H\left(\kappa_{x}, \kappa_{z}, \omega\right) \hat{\boldsymbol{f}}\left(y ; \kappa_{x}, \kappa_{z}, \omega\right) \\
& =\sum_{j=1}^{\infty} \sigma_{j}\left(\kappa_{x}, \kappa_{z}, \omega\right) a_{j}\left(\kappa_{x}, \kappa_{z}, \omega\right) \hat{\boldsymbol{\psi}}_{j}\left(y ; \kappa_{x}, \kappa_{z}, \omega\right), \\
a_{j}\left(\kappa_{x}, \kappa_{z}, \omega\right) & =\int_{-1}^{1} \hat{\boldsymbol{\phi}}_{j}^{*}\left(y ; \kappa_{x}, \kappa_{z}, \omega\right) \hat{\boldsymbol{f}}\left(y ; \kappa_{x}, \kappa_{z}, \omega\right) \mathrm{d} y,
\end{aligned}
$$

where $\sigma_{1} \geqslant \sigma_{2} \geqslant \cdots>0$ denote the singular values of $H$, and the singular functions $\hat{\boldsymbol{\phi}}_{j}=\left[\hat{f}_{1 j} \hat{f}_{2 j} \hat{f}_{3 j}\right]^{\mathrm{T}}$ and $\hat{\boldsymbol{\psi}}_{j}=\left[\hat{u}_{j} \hat{v}_{j} \hat{w}_{j}\right]^{\mathrm{T}}$ are respectively the forcing and response directions corresponding to $\sigma_{j}$. In principle, there is an infinite number of singular values/modes because the wall-normal coordinate is continuous. For the discretized equation, the total number of singular values/modes is twice the number of grid points in $y$ since the resolvent operator $R_{A}$ in (2.9) acts on a vector of two functions in $y$. As highlighted by McKeon \& Sharma (2010), the singular value decomposition effectively demonstrates that there is a limited number of relatively highly amplified modes within this total number of modes. Throughout this paper, we consistently refer to $\hat{\boldsymbol{\psi}}_{j}$ as the resolvent mode, and distinguish it from the real turbulent flow that, under stationary 
conditions, can be represented by a weighted sum of the resolvent modes. The latter is denoted the weighted mode. Note that the resolvent modes were denoted response modes in McKeon \& Sharma (2010), McKeon et al. (2013) and Sharma \& McKeon (2013).

While the singular values of $H$ are unique, additional treatment is necessary to obtain unique singular functions. Unlike in a pipe, the singular values come in pairs due to the wall-normal symmetry in the channel (which reflects itself in the resolvent operator); see, for example, figure $4(a)$. For the modes with smaller streamwise and spanwise wavelengths than the channel half-height, the singular values come in equal pairs. Therefore, any linear combination of the corresponding singular functions represents a legitimate singular function. For example, if the symmetric and antisymmetric modes are denoted by $\psi_{s}$ and $\psi_{a}$ where $\left|\psi_{s}\right|=\left|\psi_{a}\right|$, the singular function given by $\psi_{d}=\psi_{s}-\psi_{a}$ is zero in one half of the channel and twice $\psi_{s}$ in the other half. Clearly, $\psi_{d}$ is also a singular function of the transfer function with the same singular value as $\psi_{s}$ and $\psi_{a}$. Physically, this means that the modes with lengths and widths smaller than the channel half-height exhibit the potential to independently evolve in either half of the channel provided that they are forced with a forcing (e.g. disturbance) that is present only in one half of the channel. On the other hand, for the modes with larger wavelengths than the channel half-height, the paired singular values are different and the singular modes are either symmetric or anti-symmetric in the opposite halves of the channel. Physically, these modes represent convective global phenomena, meaning that they cannot take place independently in the opposite halves of the channel. They convect with the same magnitude in the opposite halves of the channel even though they can be of the same or opposite phase.

When the paired singular values are different, we obtain unique singular functions, modulo a complex multiplicative constant of unit magnitude, by imposing an orthonormality constraint on them:

$$
\int_{-1}^{1} \hat{\boldsymbol{\phi}}_{j}^{*}\left(y ; \kappa_{x}, \kappa_{z}, \omega\right) \hat{\boldsymbol{\phi}}_{k}\left(y ; \kappa_{x}, \kappa_{z}, \omega\right) \mathrm{d} y=\int_{-1}^{1} \hat{\boldsymbol{\psi}}_{j}^{*}\left(y ; \kappa_{x}, \kappa_{z}, \omega\right) \hat{\boldsymbol{\psi}}_{k}\left(y ; \kappa_{x}, \kappa_{z}, \omega\right) \mathrm{d} y=\delta_{j k},
$$

where $\delta$ denotes the Kronecker delta. In the case where the paired singular values are equal, we impose a symmetry/anti-symmetry constraint on the singular functions in addition to the above orthonormality constraint. In other words, the corresponding singular functions assume the same magnitude throughout the channel while being in phase in one half of the channel and out of phase in the other half.

In this study, we select the unknown multiplicative constant (after orthonormalization) such that $\hat{u}_{j}\left(y_{\max } ; \kappa_{x}, \kappa_{z}, \omega\right)$ is a real number at the wall-normal location $y_{\max }$ where the absolute value of $\hat{u}_{j}$ is the largest. This choice places the maximum of $u_{j}\left(x, y, z, t ; \kappa_{x}, \kappa_{z}, \omega\right)$ at the origin $x=z=t=0$. The channel symmetries in the streamwise and spanwise directions can be used to obtain $u_{j}, v_{j}$, and $w_{j}$ in the physical domain:

$$
\begin{gathered}
u_{j}\left(x, y, z, t ; \kappa_{x}, \kappa_{z}, \omega\right)=4 \cos \left(\kappa_{z} z\right) \operatorname{Re}\left(\hat{u}_{j}\left(y ; \kappa_{x}, \kappa_{z}, \omega\right) \mathrm{e}^{\mathrm{i}\left(\kappa_{x} x-\omega t\right)}\right), \\
v_{j}\left(x, y, z, t ; \kappa_{x}, \kappa_{z}, \omega\right)=4 \cos \left(\kappa_{z} z\right) \operatorname{Re}\left(\hat{v}_{j}\left(y ; \kappa_{x}, \kappa_{z}, \omega\right) \mathrm{e}^{\mathrm{i}\left(\kappa_{x} x-\omega t\right)}\right), \\
w_{j}\left(x, y, z, t ; \kappa_{x}, \kappa_{z}, \omega\right)=-4 \sin \left(\kappa_{z} z\right) \operatorname{Im}\left(\hat{w}_{j}\left(y ; \kappa_{x}, \kappa_{z}, \omega\right) \mathrm{e}^{\mathrm{i}\left(\kappa_{x} x-\omega t\right)}\right),
\end{gathered}
$$



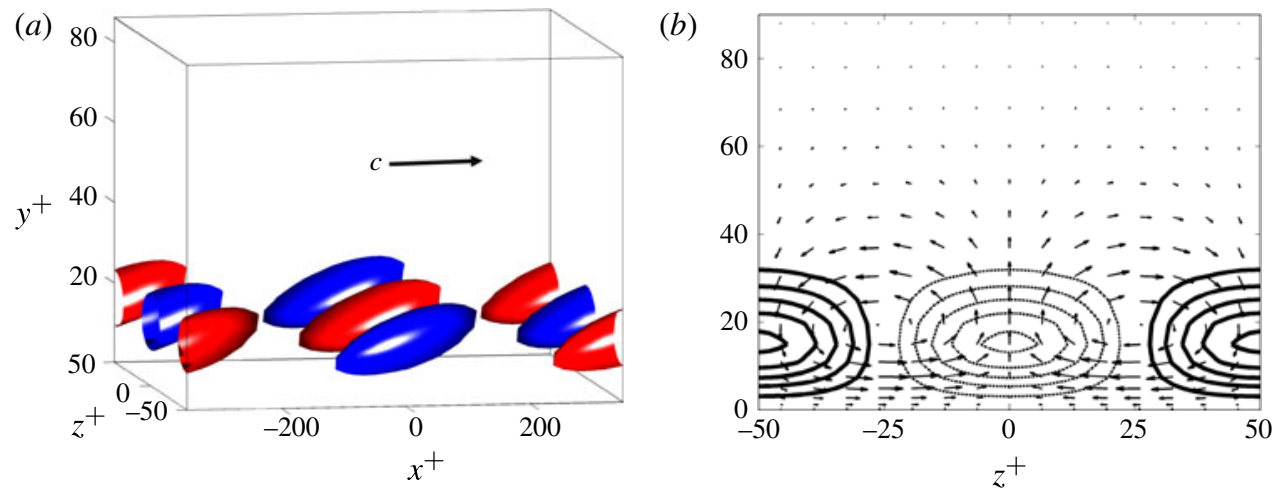

FIGURE 3. (Colour online) The principal velocity response $\boldsymbol{\psi}_{1}\left(x, y, z, t ; \kappa_{x}, \kappa_{z}, c\right)=$ $\left[\begin{array}{lll}u_{1} & v_{1} & w_{1}\end{array}\right]^{\mathrm{T}}$ for $\lambda_{x}^{+}=700, \lambda_{z}^{+}=100, c=10$, and $R e_{\tau}=10000$ at $t=0$. (a) The isosurfaces of the streamwise velocity, $u_{1}$, at $60 \%$ of its maximum. $(b)$ The streamwise velocity $\left(u_{1}\right.$, contours) and the spanwise and wall-normal velocity $\left(v_{1}, w_{1}\right.$, arrows) at $x^{+}=\lambda_{x}^{+} / 2$. The contours in $(b)$ represent positive (thick solid) and negative (thin dashed) values from 3 to 15 with increments of 3 .

where Re and Im denote the real and imaginary parts of a complex number. The representation of the forcing directions in the physical domain is obtained using similar expressions.

From the singular value decomposition (2.10) and the orthonormality constraints (2.11) it follows that if the forcing is aligned in the $\hat{\boldsymbol{\phi}}_{j}$-direction with unit energy, the response is aligned in the $\hat{\boldsymbol{\psi}}_{j}$-direction with energy $\sigma_{j}^{2}$. Consequently, the forcing and response directions with the largest gain correspond to the principal singular functions $\hat{\boldsymbol{\phi}}_{1}$ and $\hat{\boldsymbol{\psi}}_{1}$. For any $\left(\kappa_{x}, \kappa_{z}, \omega\right)$, the singular functions of $H$ should be thought of as propagating waves in the physical domain. In the rest of the paper, the resolvent modes are characterized by $c$ instead of $\omega$ and we note that prescribing any two of $\kappa_{x}$, $\omega$, and $c$ leads to the other.

Equivalent near-wall structures to those reported for pipe flows by McKeon \& Sharma (2010) and McKeon et al. (2013) are obtained for channel flows. For example, the principal singular function $\psi_{1}\left(x, y, z, t ; \kappa_{x}, \kappa_{z}, \omega\right)=\left[\begin{array}{lll}u_{1} & v_{1} & w_{1}\end{array}\right]^{\mathrm{T}}$ for the propagating wave corresponding to the energetic near-wall cycle $\left(\lambda_{x}^{+}=700, \lambda_{z}^{+}=100\right.$, $c=U\left(y^{+}=15\right)=10$ ) for $R e_{\tau}=10000$ is shown in figure 3. The streamwise component of these structures contains regions of fast- and slow-moving fluids that are aligned in the streamwise direction, slightly inclined to the wall, and are sandwiched between counter-rotating vortical motions in the cross-stream plane.

\subsection{Low-rank nature of $H$}

The operator $H$, acting on functions of $y$, can be described as low rank if a significant portion of its response to a broadband forcing in $y$ is captured by projection on the first few response directions. McKeon \& Sharma (2010) highlighted the low-rank nature of $H$ for turbulent pipe flow. Figure 4(a) shows the first twenty singular values of $H$ for $\lambda_{x}^{+}=700, \lambda_{z}^{+}=100$, and $c=10$ in turbulent channel flow with $\operatorname{Re}_{\tau}=2003$. We see that the largest pair of singular values is approximately one order of magnitude larger than the other singular values.

The energetic contribution of the $k$ th-direction $\hat{\boldsymbol{\psi}}_{k}$ to the total response in the model subject to broadband forcing in $y$ with fixed $\lambda_{x}, \lambda_{z}$, and $c$ is quantified by 

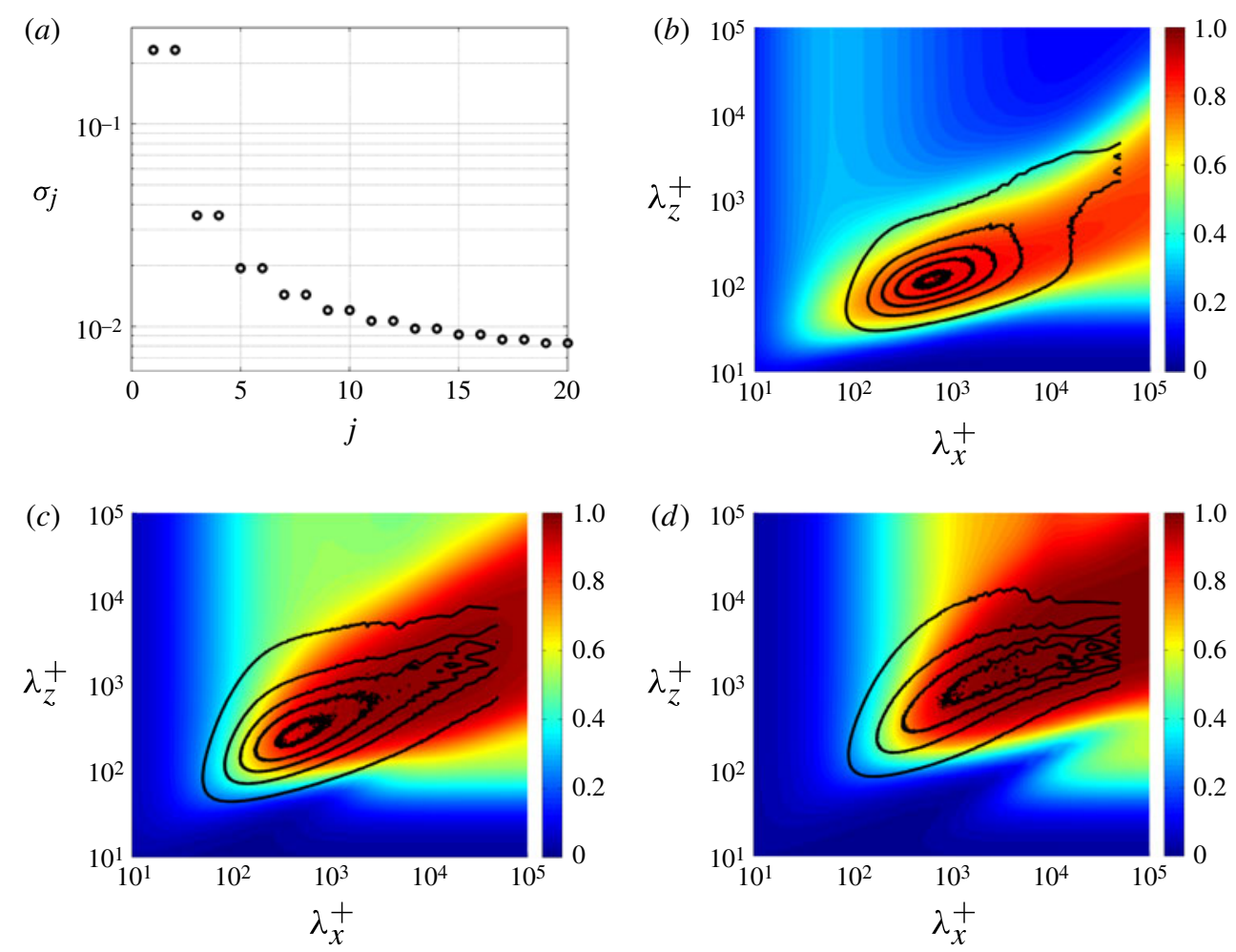

Figure 4. (a) The twenty largest singular values of $H$ for $\lambda_{x}^{+}=700, \lambda_{z}^{+}=100, c=10$, and $R e_{\tau}=2003$. $(b-d)$ The energy that is contained in the largest two response modes relative to the total response, $\left(\sigma_{1}^{2}+\sigma_{2}^{2}\right) /\left(\sum_{j=1}^{\infty} \sigma_{j}^{2}\right)$, for different streamwise and spanwise wavelengths and: $(b) c=U\left(y^{+}=15\right)$; $(c) c=U\left(y^{+}=100\right)$; and $(d) c=U(y=0.2)$. The black contours show the turbulent kinetic energy spectrum from the DNS of Hoyas \& Jiménez (2006) at the corresponding critical wall-normal locations: $(b) y^{+}=15 ;(c) y^{+}=100$; and $(d) y=0.2$. The contours represent $10 \%$ to $90 \%$ of the maximum energy spectrum at each wall-normal location with increments of $20 \%$.

$\sigma_{k}^{2} /\left(\sum_{j=1}^{\infty} \sigma_{j}^{2}\right)$. Figures $4(b)-4(d)$ highlight the low-rank nature of $H$ by showing that the first two principal response directions $\hat{\psi}_{1}$ and $\hat{\psi}_{2}$ contribute more than $80 \%$ of the total response over a large range of wall-parallel wavelengths (red region) for wave speeds $c=U\left(y^{+}=15\right), U\left(y^{+}=100\right), U(y=0.2)$, and $R e_{\tau}=2003$. The relevance of studying the low-rank approximation of $H$ is further emphasized by noting that the most energetic wavenumbers from the DNS of Hoyas \& Jiménez (2006) (contours) coincide with the wavenumbers and critical wave speeds for which $H$ is low rank. We note that the streamwise velocity has the largest contribution to the kinetic energy. Even though the shapes of the two-dimensional wall-normal and spanwise spectra may be significantly different from the streamwise spectrum, the contours corresponding to $70 \%$ of the maximum in all spectra (not shown) lie within the region where the contribution of the largest two singular values is more than $50 \%$.

\subsection{Rank-1 model subject to broadband forcing}

In the present study, we consider a rank-1 model by only keeping the most energetic forcing and response directions corresponding to $\sigma_{1}$ and show that significant 
understanding of the scaling of wall turbulence can be obtained using this simple model. This is motivated by the observation in $\S 2.3$ that the operator $H$ is essentially a directional amplifier. In other words, we expect to see the principal singular response of $H$ in real turbulent flows provided that the principal forcing direction is present in the nonlinear forcing term. Even though the resolvent modes corresponding to $\sigma_{1}$ and $\sigma_{2}$ comparably contribute to the total response, cf. $\S 2.3$, considering one of the resolvent modes is sufficient for capturing the wall-normal shape of the energy density. This is because the two resolvent modes are symmetric/anti-symmetric counterparts of each other and have the same magnitude. Therefore, accounting for both resolvent modes yields the same result as accounting for one resolvent mode.

It is well-known that the streamwise energy spectrum can be divided into regions that scale in inner and outer variables (see, for example, Morrison et al. 2004). Our objective is to explore the Reynolds-number scaling of the streamwise energy density and predict the behaviour of the streamwise turbulence intensity at high $\operatorname{Re}_{\tau}$. We focus on the streamwise velocity because it dominates the kinetic energy density in turbulent flows. Similarly, the principal singular responses of $H$ that result in the largest energy amplification are dominated by their streamwise component, such that the proposed gain-based decomposition yields the streamwise velocity most accurately. This is in agreement with previous linear analyses of the global optimal responses, e.g. del Álamo \& Jiménez (2006) and Hwang \& Cossu (2010). We note that higher-order resolvent modes may have comparable or larger wall-normal and spanwise components relative to the streamwise velocity, the study of which is a subject of ongoing work.

In order to use the smallest number of assumptions, we consider the case where the forcing $\hat{f}$ equals the principal forcing direction $\hat{\boldsymbol{\phi}}_{1}$. Consequently, the forcing has unit energy for all wave parameters, meaning that it is broadband in $\kappa_{x}, \kappa_{z}$, and $c$. For the rank-1 model with broadband forcing, we define the premultiplied streamwise energy density of the principal response of $H$ by

$$
E_{u u}\left(y ; \kappa_{x}, \kappa_{z}, c\right)=\kappa_{x}^{2} \kappa_{z}\left(\sigma_{1}\left(\kappa_{x}, \kappa_{z}, c\right)\left|u_{1}\right|\left(y ; \kappa_{x}, \kappa_{z}, c\right)\right)^{2},
$$

such that the premultiplied one-dimensional energy densities and the energy intensity are obtained by integrating $E_{u u}\left(y ; \kappa_{x}, \kappa_{z}, c\right)$ over the set of all wave parameters $\mathscr{S}$, e.g.

$$
\begin{aligned}
E_{u u}(y, c) & =\iint_{\mathscr{S}} E_{u u}\left(y ; \kappa_{x}, \kappa_{z}, c\right) \mathrm{d} \log \left(\kappa_{x}\right) \mathrm{d} \log \left(\kappa_{z}\right), \\
E_{u u}(y) & =\iiint_{\mathscr{S}} E_{u u}\left(y ; \kappa_{x}, \kappa_{z}, c\right) \mathrm{d} \log \left(\kappa_{x}\right) \mathrm{d} \log \left(\kappa_{z}\right) \mathrm{d} c,
\end{aligned}
$$

and $E_{u u}\left(y, \kappa_{x}\right)$ and $E_{u u}\left(y, \kappa_{z}\right)$ are determined similarly.

The above formulation of the energy density is used in $\S 3$ to identify the contribution of confined subsets of wave parameters to the energy density. We establish that the energy density exhibits universal behaviour with $R e_{\tau}$ for properly selected subsets of wave parameters. It is further shown that the emerging scales are consistent with those observed in experiments. In addition, the scales of energetically dominant waves roughly agree with the scales of dominant near-wall motions in real turbulent flows.

\subsection{Computational approach}

A pseudo-spectral method is used to discretize the differential operators in the wallnormal direction on a set of Chebyshev collocation points. This is implemented using the Matlab differentiation matrix Suite developed by Weideman \& Reddy (2000). 


$\begin{array}{lcccccccccc}R e_{\tau} & N_{x} & N_{y} & N_{z} & N_{c} & \lambda_{x, \min }^{+} & \lambda_{x, \max } & y_{\min }^{+} & \lambda_{z, \min }^{+} & \lambda_{z, \max } & U_{c l} \\ 934 & 64 & 251 & 32 & 100 & 10 & 10^{6} & 0.07 & 10 & 100 & 22.39 \\ 2003 & 64 & 251 & 32 & 100 & 10 & 5 \times 10^{5} & 0.15 & 10 & 50 & 24.02 \\ 3333 & 64 & 301 & 32 & 100 & 10 & 3 \times 10^{5} & 0.18 & 10 & 30 & 25.22 \\ 10000 & 64 & 401 & 32 & 100 & 10 & 10^{5} & 0.3 & 10 & 10 & 27.81 \\ 30000 & 80 & 601 & 40 & 100 & 10 & 3.3 \times 10^{6} & 0.4 & 10 & 33 & 30.39\end{array}$

TABLE 1. Summary of the selected parameters in numerical computations at different Reynolds numbers. In the wall-normal direction, $N_{y}$ Chebyshev collocation points are used with $y_{\min }^{+}$denoting the closest point to the wall. In the streamwise and spanwise directions, $N_{x}$ and $N_{z}$ logarithmically spaced wavelengths are used between $\lambda_{\min }^{+}$and $\lambda_{\max }$. In addition, $N_{c}$ linearly spaced wave speeds are chosen between $c_{\min }=2$ and $c_{\max }=U_{c l}$.

Table 1 summarizes the selected range of wave parameters and their respective resolution in numerical computations. It has been verified that the excluded wave parameters are not energetically important and therefore do not change the results of the present study.

An efficient randomized scheme developed by Halko, Martinsson \& Tropp (2011) is utilized to compute the principal singular directions of $H$ for different Reynolds numbers and wave parameters. The accuracy and computation time depend on the decay of the singular values; a faster decay results in high accuracy or equivalently less computation time to reach the same accuracy. In addition, if the singular values are not well separated, the problem of computing the associated singular functions is badly conditioned, meaning that it is hard for any method to determine them very accurately. In this study, the above scheme approximately halves the total computation time relative to Matlab's svds algorithm. This becomes increasingly important considering the three-dimensional wave parameter space that we need to explore and the large size of the discretized resolvent operator (twice the number of collocation points in $y$ ) at high Reynolds numbers. In addition, the randomized nature of this scheme enables its parallel implementation which makes it especially suitable for large-scale computations. Even though we have not used this feature in the present study, it may find use in designing turbulent flow control strategies, e.g. by means of spatially or temporally periodic actuations.

\section{Universal behaviour of the resolvent}

The formulation of $\S 2$ facilitates analysis of the contribution of different wave parameters $\left(\kappa_{x}, \kappa_{z}, c\right)$ to the streamwise energy density. For the rank-1 model with broadband forcing, the energy density of each wave is determined from the principal singular values and singular functions of the transfer function $H$; see (2.13). In this section, we identify unique classes of wave parameters for which $E_{u u}\left(y ; \kappa_{x}, \kappa_{z}, c\right)$ exhibits either universal behaviour with $\operatorname{Re}_{\tau}$ or geometrically self-similar behaviour with distance from the wall. Each class is characterized by a unique range of wave speeds and a unique scaling of the wall-normal coordinate and the wall-parallel wavelengths. These classes are inherent to the linear mechanisms in the NSE and are rigorously identified by analysis of the transfer function. 

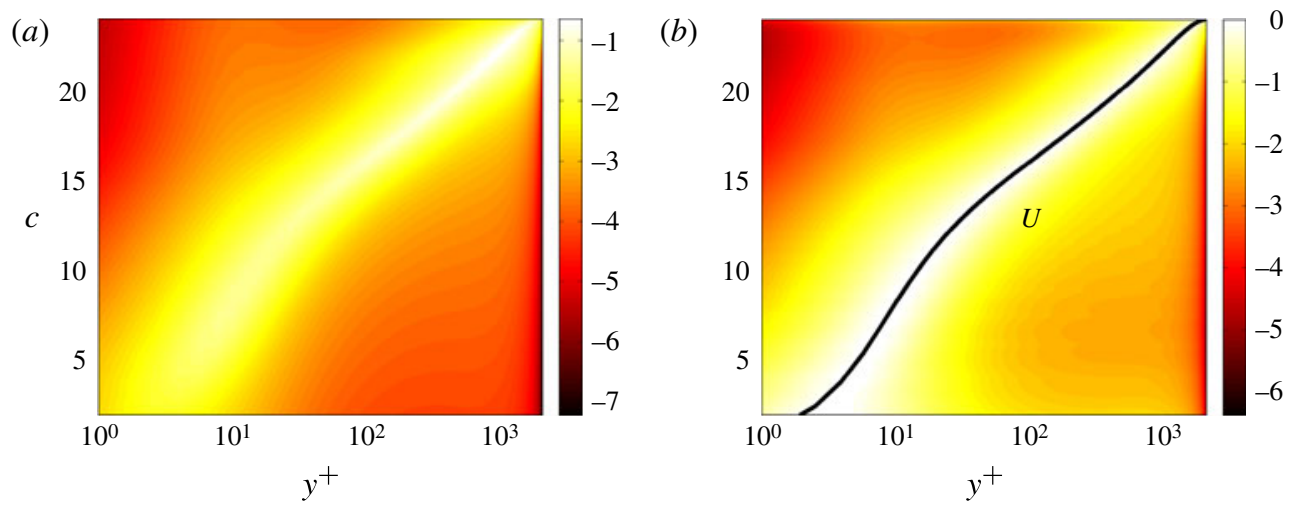

FIGURE 5. (Colour online) (a) The one-dimensional energy density $\operatorname{Re}_{\tau}^{-2} E_{u u}(y, c)$ for $\mathscr{S}=\mathscr{S}_{e}$ and $R e_{\tau}=2003$; and $(b)$ the energy density normalized by its maximum value over all $y$ for fixed values of $c$. The colours are in logarithmic scale. The turbulent mean velocity is shown by the black curve in $(b)$.

\subsection{Requirement for universality of the resolvent modes}

We start by showing that a requirement for universal behaviour is the wall-normal locality of the resolvent modes. This is done by examining the underlying operators in $H$, cf. (2.7)-(2.9). We see that the difference between the turbulent mean velocity and the wave speed, $U(y)-c$, and its wall-normal derivatives, $U^{\prime}(y)$ and $U^{\prime \prime}(y)$, appear as spatially varying coefficients in $H$. Since the turbulent mean velocity scales differently with $R e_{\tau}$ in different wall-normal locations, only the resolvent modes that are sufficiently narrow in $y$ have the potential to be universal. This is because such resolvent modes are purely affected by a certain part of the mean velocity that scales uniquely with $R e_{\tau}$.

We next show that the resolvent modes corresponding to the energetically significant modes are in fact localized. As summarized by LeHew, Guala \& McKeon (2011), the energetic contribution of structures with convection velocities less than $10 u_{\tau}$ and larger than the centreline velocity $U_{c l}=U(y=1)$ is negligible in real turbulent flows. However, we are interested in determining the effect of a broader range of wave speeds on the energy density. Note that small values of $c$ result in small amplification because the corresponding singular values are small. In fact, it is shown in $\S 4$ that including the modes with $c \lesssim 2$ does not improve the matching error between the model-based and DNS-based energy intensities. This motivates defining a conservative subset of $\mathscr{S}$, denoted by $\mathscr{S}_{e}$, that includes all wall-parallel wavenumbers and the energetically important wave speeds:

$$
\mathscr{S}_{e}=\left\{\left(\kappa_{x}, \kappa_{z}, c\right) \mid 2 \leqslant c \leqslant U_{c l}\right\} .
$$

Figure 5 shows the one-dimensional energy density as a function of wave speed $E_{u u}(y, c)$ for $\operatorname{Re}_{\tau}=2003$ and $\mathscr{S}=\mathscr{S}_{e}$. As evident from figure 5(a), the energy density for a fixed $c$ is localized in a narrow wall-normal region; note that the colours are given in logarithmic scale. The localization is highlighted in figure $5(b)$ where $E_{u u}(y, c)$ is normalized by its maximum value over $y$ for fixed values of $c$. We see that the largest energy amplification takes place in the vicinity of the critical wall-normal location where the turbulent mean velocity (thick black curve) equals the wave speed. McKeon \& Sharma (2010) argued that emergence of critical layers is one of the three 
means of maximizing the Hilbert-Schmidt norm of $H$ (sum of squares of the singular values), i.e. by locally minimizing the term $U(y)-c$ that appears in the resolvent operator $R_{A}$ given in (2.9).

According to Taylor's frozen-turbulence hypothesis (Taylor 1938), the flow structures in boundary layers propagate downstream with a speed close to the local mean velocity. Consistent with this hypothesis, figure $5(b)$ shows that among all the waves with arbitrary streamwise and spanwise wavelengths at the wall-normal location $y$, the ones with critical speed $c=U(y)$ are the most highly amplified. This provides strong evidence for the importance of critical layers in amplification of flow disturbances. In addition, figure $5(b)$ shows that the scatter in the energetic wave speeds increases as the peak of energy density approaches the wall. This agrees with the practical observation that Taylor's hypothesis yields inaccurate energy spectra close to the wall; see, for example, Kim \& Hussain (1993), del Álamo \& Jiménez (2009), Monty \& Chong (2009) and LeHew et al. (2011).

\subsection{Requirement for geometric self-similarity of the resolvent modes}

We show that a necessary condition for existence of geometrically self-similar resolvent modes is the presence of a logarithmic region in the turbulent mean velocity. The boundary conditions in the inhomogeneous direction $y$, the wall-normal symmetry relative to the centreplane, and the presence of $y$-dependent coefficients, e.g. $U(y)-c$, in the resolvent pose limitations on wall-normal scaling of the transfer function. As discussed later in $\S 3.3$, the first two limitations are removed owing to the critical behaviour of the resolvent modes, cf. §3.1, requiring that the resolvent modes have a zero support near the walls and the centreplane. The third limitation concerns scalability of $U(y)-c, U^{\prime}(y)$, and $U^{\prime \prime}(y)$ in the resolvent, cf. (2.9), and reduces to identifying the necessary conditions under which

$$
U(y)-c=g_{1}\left(y / y_{c}\right),
$$

for some functions $U(y)$ and $g_{1}(y)$ and some scale $y_{c}$ to be determined. Let the relationship between $c$ and $y_{c}$ be governed by $c=g_{2}\left(y_{c}\right)$. Then, we seek the functions $U, g_{1}, g_{2}$, and the scale $y_{c}$ such that

$$
U(y)-g_{2}\left(y_{c}\right)=g_{1}\left(y / y_{c}\right) .
$$

It follows from (3.3) that $g_{2}(y)=U(y)-g_{1}(1), g_{1}(y)=U(y)-g_{2}(1)$, and $g_{2}(1)=$ $U(1)-g_{1}(1)$. Therefore, (3.3) can be rewritten as $U(y)-\left(U\left(y_{c}\right)-g_{1}(1)\right)=$ $U\left(y / y_{c}\right)-\left(U(1)-g_{1}(1)\right)$, or $U(y)-U\left(y_{c}\right)=U\left(y / y_{c}\right)-U(1)$. The only functions that satisfy this constraint are the constant function and the logarithmic function and we have

$$
U(y)=d_{1}+d_{2} \log _{d_{3}}(y), \quad c=U\left(d_{4} y_{c}\right),
$$

where $d_{1}$ to $d_{4}$ are constants. The wall-normal scale corresponds to the wall-normal location where $c=U\left(d_{4} y_{c}\right)$. The constant $d_{4}$ is arbitrary since it enters as a coefficient in front of the scale $y_{c}$. We select $d_{4}=1$ such that $y_{c}$ is the critical wall-normal location corresponding to the wave speed $c$. Therefore, in the presence of a logarithmic mean velocity, the height of the resolvent modes scales with $y_{c}$.

\subsection{Universal modes and self-similar modes}

We start by reviewing the universal behaviour of the turbulent mean velocity. This is a prerequisite to studying the universality of the principal propagating waves since the latter holds for critical modes only, as discussed in $\$ 3.1$. In the commonly accepted 


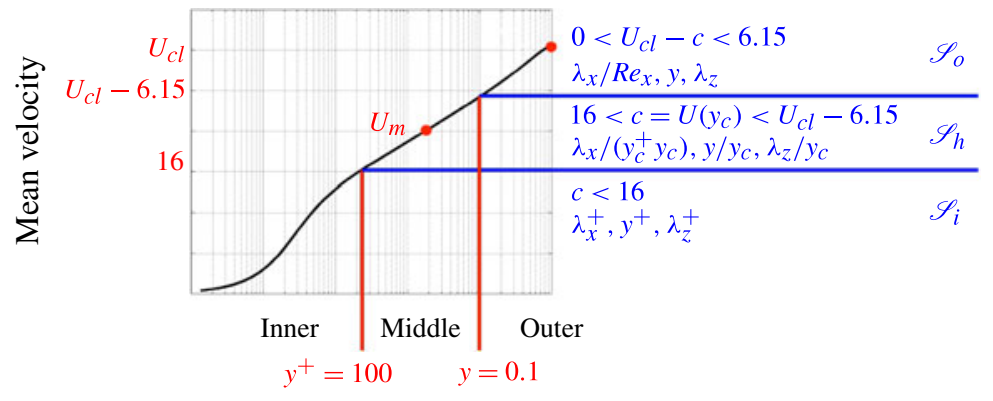

FIGURE 6. (Colour online) Schematic of the different regions of the mean velocity and the associated classes of induced scales on the propagating waves. The mean velocity is denoted by $U_{c l}$ in the centreplane and by $U_{m}$ in the geometric mean of the middle region. The inner, self-similar, and outer classes of modes are denoted by $\mathscr{S}_{i}, \mathscr{S}_{h}$, and $\mathscr{S}_{o}$, respectively. See also table 2 and figures 7 and 8.

picture (Coles 1956), the mean velocity is divided into inner, logarithmic, and outer regions:

$$
U=B\left(y^{+}\right)+(1 / \kappa) \log \left(y^{+}\right)+(2 \Pi / \kappa) W(y),
$$

where $B$ is the inner-scaled wall function, $\Pi$ is the wake factor, $W$ is the outerscaled wake function, and $\kappa$ is the von Kármán constant also appearing in (2.3). Consequently, $U-c$ is universal with $R e_{\tau}$ for certain intervals of wave speed and appropriate wall-normal scales; see figure 6. Figure $7(a)$ shows that $U\left(y^{+}\right)-c$ is universal for $y^{+} \lesssim 100$ and fixed $c \lesssim 16$ (inner region). As shown in figure $7(b)$, the function $U(y)-c$ is universal for $y \gtrsim 0.1$ and fixed defect wave speeds relative to the centreline $0 \lesssim U_{c l}-c \lesssim 6.15$, with $U_{c l}=U(y=1)$ (outer region).

The gap between the inner and outer regions of the turbulent mean velocity is bridged by a middle region between $y^{+}=100$ and $y=0.1$. There is an abundance of numerical and experimental evidence that supports the presence of a logarithmic turbulent mean velocity in this region (see, for a recent summary, Smits et al. 2011). In this study, we consider a logarithmic law throughout the middle region corresponding to $16 \leqslant U \leqslant U_{c l}-6.15$, and note that recent experiments suggest that the lower bound on the logarithmic region depends on Reynolds number: $y^{+} \sim R e_{\tau}^{1 / 2}$, see e.g. Marusic et al. (2013).

The existence, at least approximately, of a logarithmic region in $U$ satisfies the necessary conditions in $\$ 3.2$ for presence of self-similar resolvent modes. Owing to the locality of resolvent modes around the critical layer, the waves with speed $16 \leqslant c \leqslant U_{c l}-6.15$ are at least one decade away from the walls and the centreplane and the boundary effects are negligible. This eliminates the first two limitations for presence of self-similar modes, cf. $\$ 3.2$. The constants $d_{1}=5.28, d_{2}=1 / \kappa$, and $d_{3}=$ e in $U$ given by (3.4) are obtained upon direct comparison with (3.5).

Associated with each region of the mean velocity, there is a class of wave parameters for which the low-rank approximation of $H$ exhibits either universal behaviour with $R e_{\tau}$ or self-similar behaviour with distance from the wall; see tables 2 and 3 for a summary. As illustrated in figure 6, these classes are primarily distinguished by the wave speed. The identified scales represent inherent features of the linear mechanisms in the NSE and are not arbitrary: (i) the wall-normal length scale is inherited from the turbulent mean velocity at the critical layer; and (ii) the 

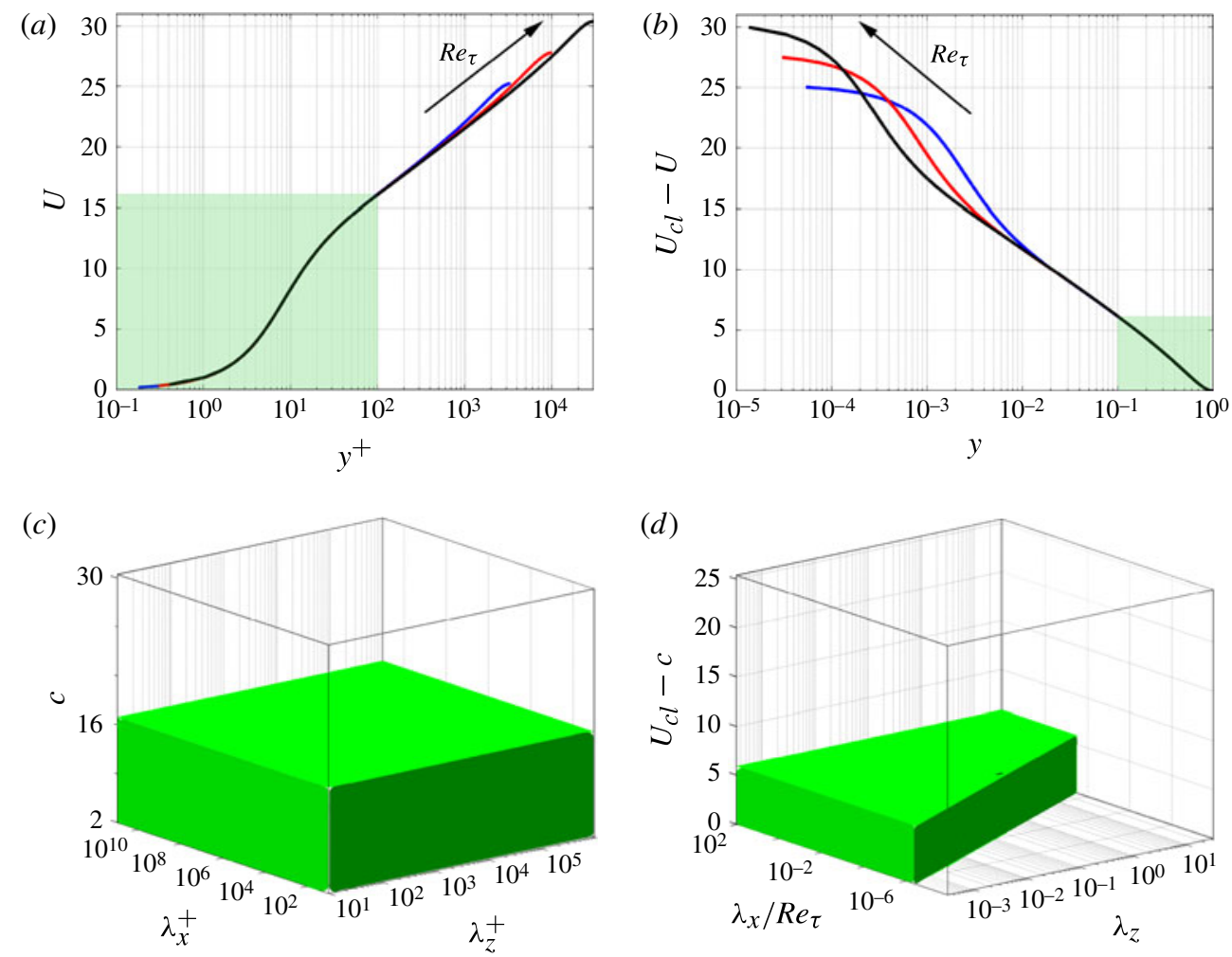

FIgURE 7. (Colour online) (a) The turbulent mean velocity $U\left(y^{+}\right)$and $(b)$ the defect velocity $U_{c l}-U(y)$ relative to the centreline, for $R e_{\tau}=3333$ (blue), $R e_{\tau}=10000$ (red), and $R e_{\tau}=30000$ (black). The arrows indicate increase in the Reynolds number. Notice that the shaded regions are invariant with $R_{\tau}$. $(c)$ The inner class $\mathscr{S}_{i}$ and $(d)$ the outer class $\mathscr{S}_{o}$ of wave parameters $\left(\lambda_{x}, \lambda_{z}, c\right)$ that induce universal behaviour with $R e_{\tau}$ on the low-rank approximation of $H . \mathscr{S}_{o}$ is obtained for $R e_{\tau}>R e_{\tau, \min }=3333$.

streamwise and spanwise length scales are determined from the balance between the viscous dissipation term, $\left(1 / R e_{\tau}\right) \Delta$, and the mean advection terms, e.g. $\mathrm{i} \kappa_{x}(U-c)$, in the resolvent in (2.9). In addition, the magnitudes of the singular values and singular functions scale uniquely in each class of wave parameters, which induces unique scales on the premultiplied streamwise energy density $E_{u u}\left(y ; \kappa_{x}, \kappa_{z}, c\right)$. Next, we separately discuss each class and refer the reader to appendices $\mathrm{A}, \mathrm{B}$ and $\mathrm{C}$ for detailed derivation of the scales.

\subsubsection{The universal inner class $\mathscr{S}_{i}$}

For wave speeds in the inner region of the turbulent mean velocity, universality of $H$ requires constant $\lambda_{x}^{+}, y^{+}, \lambda_{z}^{+}$, and $c$; cf. appendix A, table 2, and figure $7(c)$. As a result, the time $T_{c}=\lambda_{x} / c$ over which the wave convects downstream for one wavelength relative to the wall reduces with $R e_{\tau}$ and the convective frequency $\omega_{c}=2 \pi / T_{c}$ increases with $R e_{\tau}$. In other words, a truly inner scale is induced on the length, height, width, and convective time of the waves that correspond to the principal resolvent modes. Therefore, the wall-normal support of the resolvent modes in outer units linearly decreases with $R e_{\tau}$, and the unit energy constraint on the resolvent modes requires that the magnitude of the resolvent modes increase with $R e_{\tau}^{1 / 2}$. The 


\begin{tabular}{|c|c|c|c|c|c|}
\hline Class & $x$-scale & $y$-scale & $z$-scale & $t$-scale & Subset of wave parameters \\
\hline Inner & $\lambda_{x}^{+}$ & $y^{+}$ & $\lambda_{z}^{+}$ & $T_{c}^{+}$ & $\mathscr{S}_{i}: 2 \lesssim c \lesssim 16$ \\
\hline Outer & $\frac{\lambda_{x}}{R e_{\tau}}$ & $y$ & $\lambda_{z}$ & $R e_{\tau} T_{c l}$ & $\mathscr{S}_{o}:\left\{\begin{array}{l}0 \lesssim U_{c l}-c \lesssim 6.15 \\
\lambda_{x} / \lambda_{z} \gtrsim \gamma R e_{\tau} / \operatorname{Re}_{\tau, \min }\end{array}\right.$ \\
\hline Self-similar & $\frac{\lambda_{x}}{y_{c}^{+} y_{c}}$ & $\frac{y}{y_{c}}$ & $\frac{\lambda_{z}}{y_{c}}$ & - & $\mathscr{S}_{h}:\left\{\begin{array}{l}16 \lesssim c \lesssim U_{c l}-6.15 \\
c=U\left(y_{c}\right)=B+(1 / \kappa) \log \left(y_{c}^{+}\right) \\
\lambda_{x} / \lambda_{z} \gtrsim \gamma\end{array}\right.$ \\
\hline Middle & $\lambda_{x}$ & $\sqrt{y y^{+}}$ & $\sqrt{\lambda_{z} \lambda_{z}^{+}}$ & $T_{m}$ & $\mathscr{S}_{m}:\left\{\begin{array}{l}\left|U_{m}-c\right| \lesssim d \\
\lambda_{x} / \lambda_{z} \gtrsim \gamma \sqrt{R e_{\tau} / R e_{\tau, \min }}\end{array}\right.$ \\
\hline
\end{tabular}

TABLE 2. Summary of the length scales and wave speeds for the universal modes of the transfer function $H$. See also figure 6.

\begin{tabular}{|c|c|c|c|c|c|c|c|c|}
\hline Class & Subset & $\kappa_{x}$ & $(\mathrm{~d} / \mathrm{d} y)$ & $\kappa_{z}$ & $\omega_{i, o, m}$ & $\sigma_{1}$ & $u_{1}$ & $E_{u u}$ \\
\hline Inner & $\mathscr{S}_{i}$ & $R e_{\tau}$ & $R e_{\tau}$ & $R e_{\tau}$ & $R e_{\tau}$ & $R e_{\tau}^{-1}$ & $R e_{\tau}^{1 / 2}$ & $R e_{\tau}^{2}$ \\
\hline Outer & $\mathscr{S}_{0}$ & $R e_{\tau}^{-1}$ & 1 & 1 & $R e_{\tau}^{-1}$ & $R e_{\tau}^{2}$ & 1 & $R e_{\tau}^{2}$ \\
\hline Self-similar & $\mathscr{S}_{h}$ & $\left(y_{c}^{+} y_{c}\right)^{-1}$ & $y_{c}^{-1}$ & $y_{c}^{-1}$ & - & $\left(y_{c}^{+}\right)^{2} y_{c}$ & $y_{c}^{-1 / 2}$ & $R e_{\tau}^{2}$ \\
\hline Middle & $\mathscr{S}_{m}$ & 1 & $R e_{\tau}^{1 / 2}$ & $R e_{\tau}^{1 / 2}$ & 1 & $R e_{\tau}^{1 / 2}$ & $R e_{\tau}^{1 / 4}$ & $R e_{\tau}^{2}$ \\
\hline
\end{tabular}

TABLE 3. Summary of the growth/decay rates (with respect to $R e_{\tau}$ or $y_{c}$ ) of the wallparallel wavenumbers, the wall-normal derivative, the convective frequency, the principal singular value and the principal streamwise singular function of $H$, and the premultiplied three-dimensional streamwise energy density for the classes of universal waves outlined in table 2 .

number of these waves per unit wall-parallel area and time increases with $\operatorname{Re}_{\tau}^{3}$ as their length, width, and convective time decrease with $R e_{\tau}$. Since the singular values of $H$ linearly decrease with $\operatorname{Re}_{\tau}$, the overall result is that $E_{u u}\left(y ; \kappa_{x}, \kappa_{z}, c\right)$ increases with $\operatorname{Re}_{\tau}^{2}$; cf. table 3 .

\subsubsection{The universal outer class $\mathscr{S}_{0}$}

For wave speeds close to the centreline, universality of $H$ requires constant $\lambda_{x} / R e_{\tau}$, $y, \lambda_{z}$, and $U_{c l}-c$, such that an aspect-ratio constraint $\lambda_{x} / \lambda_{z} \gtrsim \gamma R e_{\tau} / \operatorname{Re}_{\tau, \min }$ is satisfied (a conservative value for $\gamma$ is $\sqrt{10})$; cf. appendix B, table 2, and figure $7(d)$. As a result, the time $T_{c l}=\lambda_{x} /\left(U_{c l}-c\right)$ over which the wave convects upstream for one wavelength relative to an observer with speed $U_{c l}$ increases with $\operatorname{Re}_{\tau}$ and the convective frequency $\omega_{c l}=2 \pi / T_{c l}$ decreases with $R e_{\tau}$. In addition, the aspect ratio $\lambda_{x} / \lambda_{z}$ of the universal waves increases as $R e_{\tau}$. This explains why universality for this class holds for the waves with aspect ratios larger than a threshold: As the aspect ratio of the resolvent modes increases with Reynolds number, the Laplacian operator in the resolvent becomes independent of $\kappa_{x}$. Therefore, the necessary condition for the Laplacian to be universal with $R e_{\tau}$ is that $\kappa_{z}$ dominates $\kappa_{x}$ even for the smallest Reynolds number $R e_{\tau, \min }$ that is considered. This poses the above-mentioned aspect-ratio constraint on the universal waves. The magnitude of resolvent modes is independent of $R e_{\tau}$ since the resolvent modes scale with outer units in the wall-normal direction. The number of waves per unit area and time decreases with $R e_{\tau}^{2}$ since their 
length and convective time increase with $R e_{\tau}$. The singular values increase with $R e_{\tau}^{2}$ and the overall result is that the energy density $E_{u u}\left(y ; \kappa_{x}, \kappa_{z}, c\right)$ increases with $R e_{\tau}^{2}$; cf. table 3.

The waves in the outer class asymptotically approach the streamwise-constant fluctuations, i.e. $\kappa_{x}=0$, as $R e_{\tau}$ increases. These infinitely long fluctuations exhibit the largest linear transient growth in response to initial perturbations in laminar (Gustavsson 1991; Butler \& Farrell 1992; Reddy \& Henningson 1993) and turbulent (Butler \& Farrell 1993; del Álamo \& Jiménez 2006; Pujals et al. 2009) flows. In addition, they are the most highly amplified by the linear dynamics in laminar (Farrell \& Ioannou 1993b; Bamieh \& Dahleh 2001; Jovanović \& Bamieh 2005) and turbulent (Hwang \& Cossu 2010) flows subject to stochastic disturbances.

The effect of Reynolds number on the streamwise-constant fluctuations has been studied in laminar flows. For example, Gustavsson (1991) showed that the peak of linear transient growth scales with the square of centreline Reynolds number $R e_{c l}=U_{c l} h / \nu$. For the flow subject to harmonic disturbances, Jovanović \& Bamieh (2005) showed that the singular values of $H$ increase as $R e_{c l}^{2}$ when the temporal frequency $\omega$ linearly decreases with $R e_{c l}$. No other scales for the singular values were found since the laminar mean velocity $U / U_{c l}=2 y-y^{2}$ is universal with Reynolds number throughout the channel. Our study shows that the singular values in the turbulent flow increase quadratically with $R e_{\tau}$ for the waves with defect speeds, $U_{c l}-c \lesssim 6.15$, and streamwise wavelengths that linearly increase with $R e_{\tau}$, i.e. $\lambda_{x} \gtrsim \gamma \lambda_{z} R e_{\tau} / \operatorname{Re}_{\tau, \text { min }}$; cf. table 3 .

\subsubsection{The geometrically self-similar class $\mathscr{S}_{h}$}

The logarithmic region of the turbulent mean velocity yields a hierarchy of geometrically self-similar resolvent modes that are uniquely parameterized by the critical wall-normal distance $y_{c}$, i.e. $c=U\left(y_{c}\right)$; see appendix C for derivation. As summarized in table 2, the height and width of the self-similar modes scale with $y_{c}$ and their length with $y_{c}^{+} y_{c}$. In addition, the self-similar modes satisfy an aspect-ratio constraint, $\lambda_{x} / \lambda_{z} \gtrsim \gamma$, where a conservative value for $\gamma$ is $\sqrt{10}$. This agrees with the observation of Hwang \& Cossu (2010) that the optimal responses were approximately similar for $\kappa_{x} \ll \kappa_{z}$. Notice that the difference between the streamwise scaling of the self-similar resolvent modes $\lambda_{x} \sim y_{c}^{+} y_{c}$ and the scaling $\lambda_{x} \sim y$ chosen in original developments of the attached-eddy hypothesis (Townsend 1976; Perry \& Chong 1982) does not contradict the philosophy of self-similar attached eddies, i.e. the resolvent modes are still self-similar.

Any hierarchy is a subset of $\mathscr{S}$ and can be described by a representative mode with $\lambda_{x, r}, \lambda_{z, r}$, and $c_{r}=U\left(y_{c_{r}}\right)$ :

$$
\mathscr{S}_{h}\left(\lambda_{x, r}, \lambda_{z, r}, c_{r}\right)=\left\{\left(\lambda_{x}, \lambda_{z}, c\right) \mid\left\{\begin{array}{ll}
\lambda_{x}=\lambda_{x, r}\left(\frac{y_{c}^{+} y_{c}}{y_{c_{r}}^{+} y_{c_{r}}}\right), & \\
\lambda_{z}=\lambda_{z, r}\left(\frac{y_{c}}{y_{c_{r}}}\right), & \frac{100}{R e_{\tau}} \leqslant y_{c_{1}} \leqslant y_{c} \leqslant 0.1 \\
c=B+(1 / \kappa) \log y_{c}^{+},
\end{array}\right\} .\right.
$$

Here, $y_{c_{1}}$ is the critical wall-normal location associated with the smallest wave speed $c_{1}$ above which the aspect-ratio constraint is satisfied, see appendix $\mathrm{C}$.

The concept of hierarchies is illustrated in figure 8(a) where the loci of wave parameters that belong to three demonstrative hierarchies with representative modes 
(a)
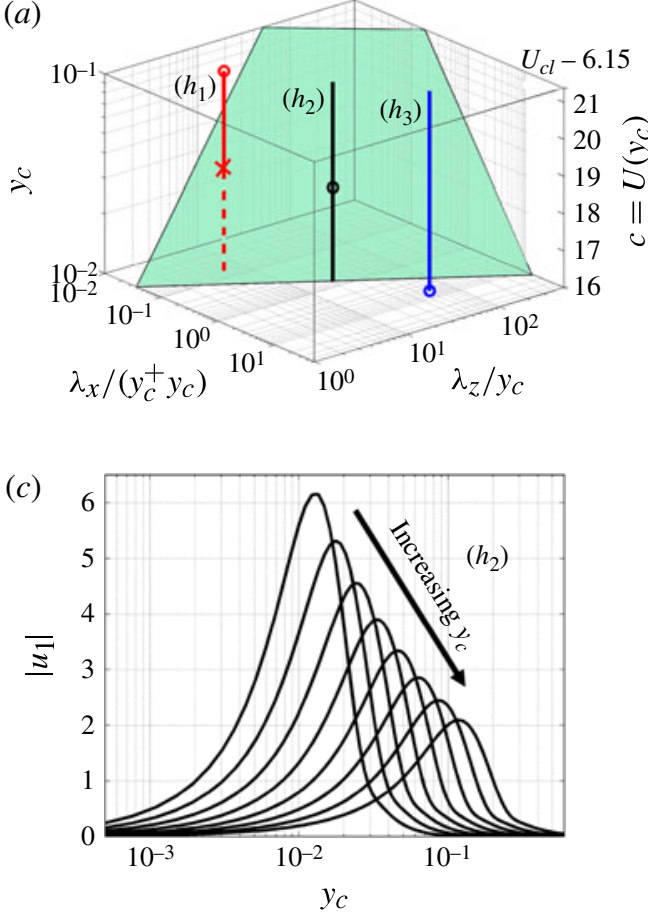

(b)

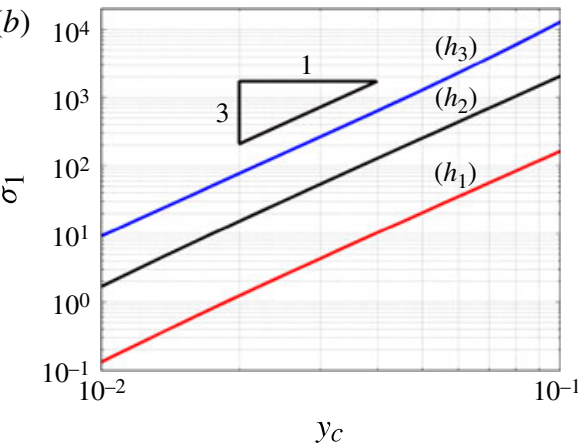

(d)

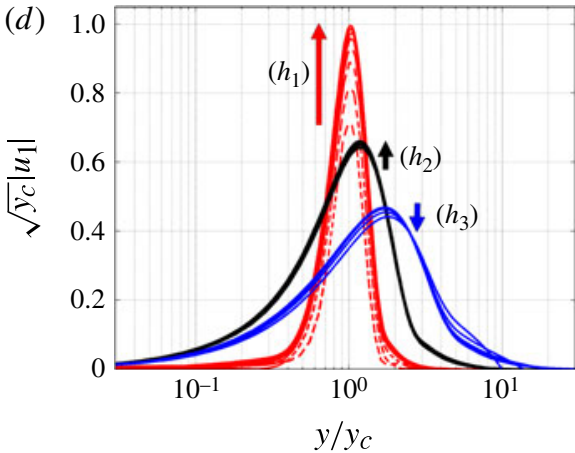

FIGURE 8. (Colour online) (a) The vertical lines are the loci of wave parameters that belong to the hierarchies with representative modes (open circles): $\kappa_{x, r}=1, \kappa_{z, r}=10$, and $c_{r}=U_{c l}-6.15\left(h_{1}\right.$, red); $(2 / 3) U_{c l}\left(h_{2}\right.$, black); and $16\left(h_{3}\right.$, blue $)$, all for $R e_{\tau}=10000$. The shaded threshold plane corresponds to the wavenumbers with aspect ratio $\lambda_{x} / \lambda_{z}=\sqrt{10}$. The modes below this plane do not belong to any hierarchy. (b) The principal singular values along the hierarchies in $(a)$. (c) The principal streamwise resolvent modes that belong to the hierarchy with $c_{r}=(2 / 3) U_{c l}\left(h_{2}\right.$, black) in $(a)$. (d) The normalized and scaled (according to table 2) principal resolvent modes along the hierarchies in $(a)$. The arrows show the direction of increasing $y_{c}$ with $100 / R e_{\tau} \leqslant y_{c} \leqslant 0.1$.

marked by open circles are shown. The mode with $\kappa_{x, r}=1, \kappa_{z, r}=10$, and $c_{r}=(2 / 3) U_{c l}\left(h_{2}\right.$, black) is representative of the very large-scale motions (McKeon $\&$ Sharma 2010). The representative modes for the other hierarchies have the same wavenumbers but different speeds, i.e. $c_{r}=16\left(h_{3}\right.$, blue $)$ and $U_{c l}-6.15\left(h_{1}\right.$, red), corresponding to the mean velocity at the upper limit of the inner region and the lower limit of the outer region. Each locus constitutes a vertical line after normalizing the length, width, and height of the modes according to the scales in (3.6) obtained from the resolvent. In fact, the resolvent modes are self-similar along any vertical line as long as $\lambda_{x} / \lambda_{z}>\gamma$. The aspect-ratio constraint requires that the wave parameters lie above the shaded threshold plane $\lambda_{x} / \lambda_{z}=\gamma$ in figure $8(a)$. For example, the waves corresponding to the dashed segment of the hierarchy with $c_{r}=U_{c l}-6.15$ do not belong to any hierarchy.

Owing to the self-similar behaviour, the principal singular values and singular functions of $H$ for all the modes in a given hierarchy can be determined from its representative mode. The principal singular values $\sigma_{1}$ corresponding to the waves that belong to the hierarchies in figure $8(a)$ are shown in figure $8(b)$. The singular values 
grow with $\left(y_{c}^{+}\right)^{2}\left(y_{c}\right)$ as theoretically predicted, cf. table 3 . Figure $8(c)$ shows the principal streamwise resolvent mode $u_{1}$ corresponding to the hierarchy with $\kappa_{x, r}=1$, $\kappa_{z, r}=10$, and $c_{r}=(2 / 3) U_{c l}$ for $100 / R e_{\tau} \leqslant y_{c} \leqslant 0.1$. The arrow shows the direction of increasing $y_{c}$. Normalizing and scaling the resolvent modes according to table 2 collapses the resolvent modes for different wave speeds; see black curves marked as $h_{2}$ in figure $8(d)$. This figure also shows the scaled resolvent modes corresponding to the hierarchies with $\kappa_{x, r}=1, \kappa_{z, r}=10$, and $c_{r}=16\left(h_{3}\right.$, blue) and $U_{c l}-6.15\left(h_{1}\right.$, red). We see that the normalized and scaled resolvent modes lie on top of each other for the hierarchy with $c_{r}=16$. For the hierarchy with $c_{r}=U_{c l}-6.15$, the resolvent modes for large $y_{c}$ collapse on each other while the resolvent modes for small $y_{c}$ are considerably different. This is expected since the aspect ratios of the modes fall below $\gamma$ as $y_{c}$ decreases. Notice that for this hierarchy, the modes corresponding to small $y_{c}$ lie below the threshold plane in figure $8(a)$.

\subsubsection{The universal middle class $\mathscr{S}_{m}$}

The Reynolds-number scaling of the self-similar class depends on the wave speed and is consistent with the inner and outer classes of resolvent modes, cf. tables 2 and 3. For example, when the wave speed is fixed as $\operatorname{Re}_{\tau}$ changes, $y_{c}^{+}$remains constant and the inner scale is recovered. When the defect wave speed $U_{c l}-c$ is fixed, $y_{c}$ remains constant and the outer scale is recovered. Consequently, the energy density corresponding to the complete range of wave speeds in the self-similar region, $16<c<U_{c l}-6.15$, is centred around the geometric mean of the middle region of the turbulent mean velocity, i.e. $y_{m}=\sqrt{10 / R e_{\tau}}$. The self-similar class is primarily concerned with geometric self-similarity of the resolvent modes. We next construct a middle class of modes $\mathscr{S}_{m}$, a subset of the self-similar class $\mathscr{S}_{h}$, with unique Reynolds-number scalings.

The wave speeds in the middle class are confined to $\left|U_{m}-c\right|<d$, with $d$ denoting a radius around $U_{m}=U\left(y_{m}\right)$. For the resolvent modes in the middle class, universality of $H$ requires constant $\lambda_{x}, \sqrt{y^{+} y}, \sqrt{\lambda_{z}^{+} \lambda_{z}}$, and $U_{m}-c$ such that the aspect-ratio constraint $\lambda_{x} / \lambda_{z} \gtrsim \gamma \sqrt{R e_{\tau} / R e_{\tau, \min }}$ is satisfied; cf. table 2 . These scales are equal to the geometric mean of the scales in the inner and outer classes, and can also be recovered from the scales of the self-similar class for fixed $\sqrt{y_{c}^{+} y_{c}}$ as $\operatorname{Re}_{\tau}$ changes. When only one Reynolds number is considered, we have $R e_{\tau, \min }=R e_{\tau}$ and the aspect-ratio constraints in the self-similar and middle classes are equivalent, i.e. the constraint $\lambda_{x} / \lambda_{z} \gtrsim \gamma \sqrt{R e_{\tau} / R e_{\tau, \min }}=\gamma$ in the middle class is the same as $\lambda_{x} / \lambda_{z} \gtrsim \gamma$ in the self-similar class. When the self-similar modes are compared across more than one Reynolds number, the aspect-ratio constraint on the middle class is more restrictive. This is because, the modes remain self-similar as the Reynolds number increases. However, they do not remain independent of $R e_{\tau}$ unless their aspect ratio is larger than $\gamma$ even for the smallest Reynolds number $R e_{\tau, \min }$ that is considered, resulting in the modified aspect-ratio constraint $\lambda_{x} / \lambda_{z} \gtrsim \gamma \sqrt{R e_{\tau} / R e_{\tau, \min }}$ for the middle class. This constraint can be obtained similarly to the constraint for the outer class, cf. $\$ 3.3 .2$ and appendix B.

The time $T_{m}=\lambda_{x} /\left|U_{m}-c\right|$ over which a wave in the middle class convects away for one wavelength relative to an observer with speed $U_{m}$ remains independent of $R e_{\tau}$ and so does the convective frequency $\omega_{m}=2 \pi / T_{m}$. These waves have the same scales as the structures in the meso-layer; see, for example, Long \& Chen (1981), Afzal (1984), Sreenivasan \& Sahay (1997) and Wei et al. (2005). The aspect-ratio constraint follows from similar arguments to those discussed for the outer class. The magnitude of the corresponding resolvent modes increases with $R e_{\tau}^{1 / 4}$ because of the 
unit-energy constraint. The number of waves per unit area and time increases with $R e_{\tau}^{1 / 2}$. In addition, the principal singular values of the waves in $\mathscr{S}_{m}$ increase with $R e_{\tau}^{1 / 2}$. The above scales result in growth of $E_{u u}\left(y ; \kappa_{x}, \kappa_{z}, c\right)$ with $R e_{\tau}^{2}$; cf. table 3.

There is a direct relationship between $\mathscr{S}_{m}$ and $\mathscr{S}_{h}$ : the union of the middle class of modes equals the union of the geometrically self-similar modes with speeds $\left|U_{m}-c\right|<d$ and aspect-ratio constraint $\lambda_{x} / \lambda_{z} \gtrsim \gamma \sqrt{R e_{\tau} / R e_{\tau, \min }}$. Since the difference between the middle class and the self-similar class becomes larger as $R e_{\tau}$ increases, our ongoing research is focused on analytical developments using the scalings of the self-similar class that bridges the gap between the inner and outer classes.

\subsection{Universality of the streamwise energy density}

We compute the streamwise energy density of the rank-1 model with broadband forcing and illustrate its universal behaviour with $R e_{\tau}$. These computations build the basis for prediction of the streamwise energy intensity at the technologically relevant values of $R e_{\tau}$ in $\S 4$. Because of the unique scales in the inner, middle, and outer classes of wave parameters, we distinguish the corresponding intervals of wave speeds by expanding the premultiplied energy density into the following three integrals:

$$
\begin{aligned}
E_{u u}\left(y, \kappa_{x}, \kappa_{z}\right) & =\int_{2}^{16} E_{u u}\left(y, \kappa_{x}, \kappa_{z}, c\right) \mathrm{d} c \\
& +\int_{16}^{U_{c l}-6.15} E_{u u}\left(y, \kappa_{x}, \kappa_{z}, c\right) \mathrm{d} c+\int_{U_{c l}-6.15}^{U_{c l}} E_{u u}\left(y, \kappa_{x}, \kappa_{z}, c\right) \mathrm{d} c .
\end{aligned}
$$

Similar expansions can be written for $E_{u u}\left(y, \kappa_{x}\right), E_{u u}\left(y, \kappa_{z}\right)$, and $E_{u u}(y)$. In spite of the different behaviour of singular values and singular functions, the energy density increases with $R e_{\tau}^{2}$ in all three classes of wave parameters. Figure 9 shows the premultiplied one-dimensional energy densities and the energy intensity confined to each class of wave parameters and normalized by $R e_{\tau}^{2}$. The same contour levels are used for all Reynolds numbers, $R e_{\tau}=3333$ (blue), 10000 (red), and 30000 (black), in figures $9(a)-9(f)$. Notice that confining the wavenumbers to $\mathscr{S}_{i}, \mathscr{S}_{m}$, and $\mathscr{S}_{o}$ yields a universal energy density as summarized in tables 2 and 3.

The inner peak of streamwise energy density in the rank-1 model with broadband forcing occurs at $y^{+} \approx 11, \lambda_{x}^{+} \approx 184$, and $\lambda_{z}^{+} \approx 44$; see figures $9(a)$ and $9(d)$. The location of the above wall-normal peak represents an integral effect over all wave parameters in $\mathscr{S}_{i}$ and corresponds to the critical speed $c \approx 8.5$. The inner peak is comparable with the location, length, and spacing of the most energetic structures associated with the near-wall cycle, i.e. $y^{+} \approx 15, \lambda_{x}^{+} \approx 700-1000$, and $\lambda_{z}^{+} \approx 100$ (see, for example, Hoyas \& Jiménez 2006). Figures $9(c)$ and $9(f)$ show that the outer peak takes place at $y \approx 0.45$ (corresponding to critical defect speed $U_{c l}-c \approx 2$ ) for $\lambda_{x} / R e_{\tau} \approx 0.1$, and $\lambda_{z} \approx 2$. This peak points to much longer structures relative to the LSM structures observed in experiments, i.e. $\lambda_{x} \approx 3$ (see, for example in boundary layers, Adrian et al. 2000). The same length scales are reported for channels and pipes (see, for example, Guala, Hommema \& Adrian 2006; Monty et al. 2009).

The middle peak takes place at $\sqrt{y y^{+}} \approx 5.2-5.8$ (corresponding to critical speed $\left.c \approx U_{m}\right), \lambda_{x} \approx 12-16$, and $\sqrt{\lambda_{z} \lambda_{z}^{+}} \approx 20$; see figures $9(b)$ and $9(e)$. It has the same streamwise and wall-normal scalings as the VLSMs and its location is comparable with the most energetic VLSMs located at $\sqrt{y y^{+}} \approx 3.9$ and $\lambda_{x} \approx 6$ in boundary layers (see, for example, Marusic et al. 2010a) and at $\lambda_{x} \approx 12-20$ in pipes and channels (see, for example, Monty et al. 2009). Spanwise correlations of experimental data show that the spanwise wavelength of modes in the middle region increases 

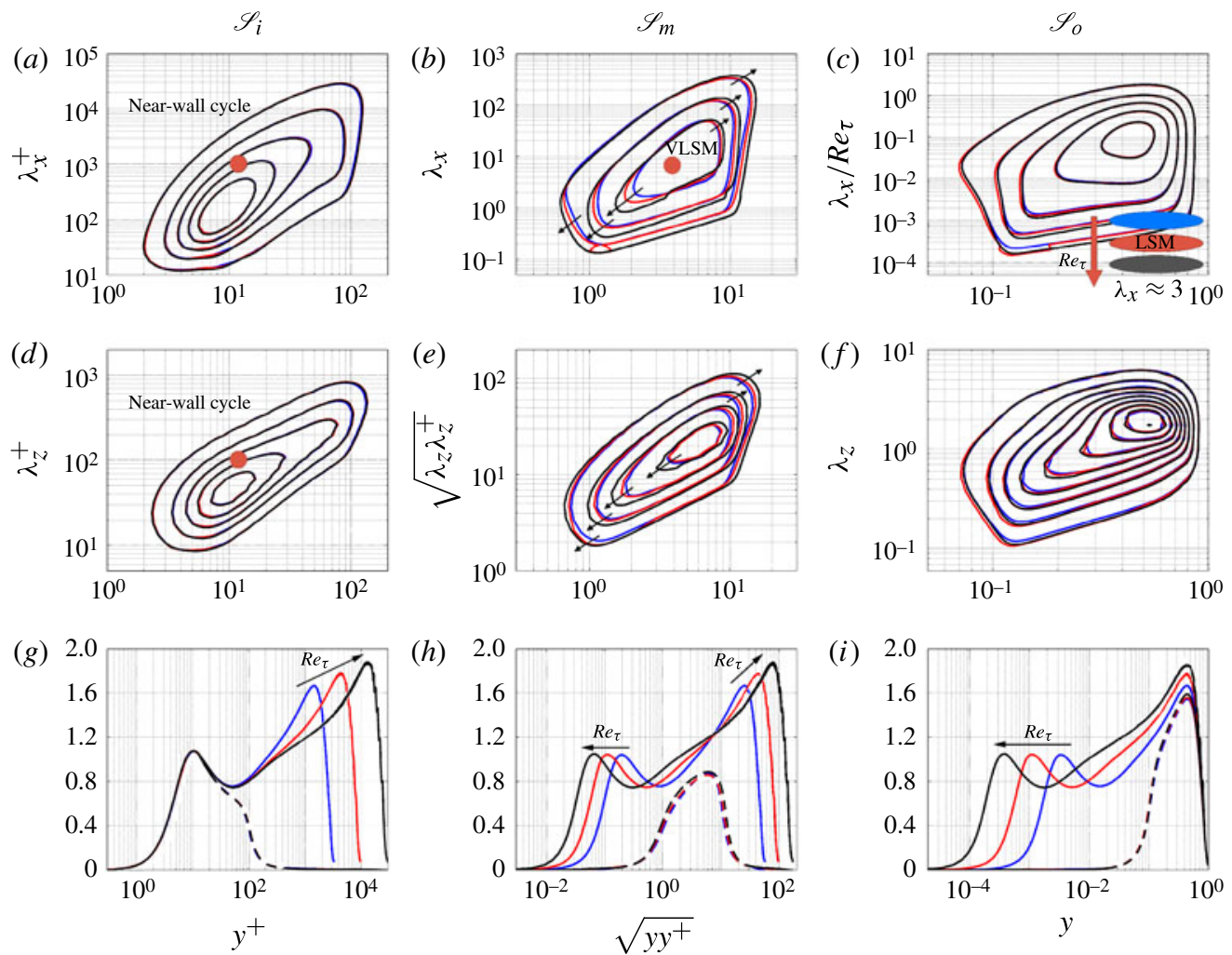

FIGURE 9. (Colour online) The premultiplied one-dimensional streamwise energy density $\operatorname{Re}_{\tau}^{-2} E_{u u}\left(y, \lambda_{x}\right)$ in $(a-c)$ and $\operatorname{Re}_{\tau}^{-2} E_{u u}\left(y, \lambda_{z}\right)$ in $(d-f)$; and the streamwise energy intensity $\operatorname{Re}_{\tau}^{-2} E_{u u}(y)$, dashed curves in $(g-i)$ for the rank-1 model with broadband forcing. The wave parameters are confined to $\mathscr{S}=\mathscr{S}_{i}$ in $(a, d, g) ; \mathscr{S}=\mathscr{S}_{m}$ in $(b, e, h)$; and $\mathscr{S}=\mathscr{S}_{o}$ in $(c, f, i)$. The Reynolds numbers are $R e_{\tau}=3333$ (blue), $R e_{\tau}=10000$ (red), and $R e_{\tau}=30000$ (black). The contour levels decrease by 0.05 from their maximum value of $0.25(a) ; 0.15(b)$; and $0.25(c)$, and by 0.1 from their maximum value of $0.5(d) ; 0.4(e)$; and $0.8(f)$. The solid curves in $(g-i)$ are obtained by confining the wave parameters to $\mathscr{S}=\mathscr{S}_{e}$. The arrows indicate increase in the Reynolds number.

approximately linearly with y, see e.g. Monty et al. (2007) and Bailey et al. (2008). The self-similar scales of the resolvent modes in the middle region are consistent with these results. The Reynolds-number scaling of the spanwise wavelength appears to still be under investigation. Since the spanwise peak of the one-dimensional spectrum is obtained by including a range of modes with speeds $\left|U_{m}-c\right|<d$ (instead of focusing on one mode), the Reynolds-number scaling of the spanwise peak is similar to the wall-normal scaling of the modes. The organization of the self-similar coherent motions in the logarithmic layer of real turbulent flows has been studied by many authors, e.g. see Tomkins \& Adrian (2003), del Álamo et al. (2006) and Flores \& Jiménez (2010). In addition, Hwang \& Cossu (2011) addressed the self-sustaining mechanisms of these coherent motions. Studying the implications of the identified scalings of the resolvent modes for these structures is a topic of future research.

In making the above comparisons, it is important to note the distinction between the resolvent modes and the real turbulent flow that can be represented by a weighted 
superposition of the resolvent modes. The agreement between the admitted scales of the principal resolvent modes and the scalings observed in real flows is striking considering the simplicity of the rank-1 model subject to broadband forcing. This agreement emphasizes the role of linear mechanisms and critical layers in determining the scaling of turbulent flows. In addition, the differences between the scalings highlights the role of nonlinearity in shaping the weights of the resolvent modes. We also note that the experimentally obtained outer peak in the two-dimensional spectrum and the wavelengths associated with VLSMs and LSMs may be contaminated by use of Taylor's hypothesis and lack of sufficient scale separation at relatively low $R e_{\tau}$.

The one-dimensional energy densities can be integrated in the remaining wallparallel direction to obtain the streamwise energy intensity $E_{u u}(y)$ for the rank-1 model with broadband forcing. The dashed curves in figures $9(g)-9(i)$ are the energy intensities normalized by $R e_{\tau}^{2}$ obtained by confining the wave parameters to $\mathscr{S}_{i}, \mathscr{S}_{m}$, and $\mathscr{S}_{o}$, respectively. As expected, the energy intensities are independent of Reynolds number when confined to the universal classes of wave parameters. The solid curves are obtained by integrating the energy density over all wavenumbers and wave speeds $2 \leqslant c \leqslant U_{c l}$, i.e. by confining the wave parameters to $\mathscr{S}_{e}$. These figures highlight the selection of two local peaks by the linear amplification mechanism where the inner and outer peaks dominate the middle peak. The inner peak occurs close to the inner peak of the streamwise intensity in real turbulent flows. While the energy intensity of real flows exhibits outer scales near the centre of the channel, there is no strong evidence for presence of an outer peak even for high $R e_{\tau}$.

As evident from figure $9(g)$, the universal inner waves contribute more than $96 \%$ of the total energy intensity for $y^{+}<20$ for all Reynolds numbers. On the other hand, figure 9(i) shows that the universal outer waves capture a smaller amount of the total intensity for $y=0.45$ as $R e_{\tau}$ increases; $95 \%$ for $R e_{\tau}=3333$ versus $86 \%$ for $R e_{\tau}=30000$. This is because the aspect-ratio constraint in $\mathscr{S}_{o}$ excludes more wavenumbers from $\mathscr{S}_{e}$ as $R e_{\tau}$ increases. The excluded waves are not universal with $R e_{\tau}$ and their contribution to the energy intensity is not completely negligible. A similar reasoning explains why the universal middle scale captures $82 \%$ of the total energy intensity at $\sqrt{y y^{+}}=\sqrt{10}$ for $R e_{\tau}=3333$ versus $72 \%$ for $R e_{\tau}=30000$; cf. figure $9(h)$.

At the end of this section, we recall that the streamwise energy densities and intensities thus far were obtained for the model with broadband forcing in $\lambda_{x}, \lambda_{z}$, and $c$. In $\S 4$, we consider a non-broadband forcing by introducing an optimally shaped energy density.

\section{Predicting the streamwise energy intensity}

In this section, we introduce a model for predicting the energy intensity of real turbulent flows by considering a non-broadband forcing in wave speed. This is done by incorporating a positive weight function $W(c)$ that amplifies or attenuates the energy density $E_{u u}(y, c)$ of the rank-1 model with broadband forcing. Even though $W(c)$ differs from a true forcing spectrum (that also depends on the wall-parallel wavelengths), it provides the model with sufficient degrees of freedom for predicting the energy intensity. In addition, since each wave speed is associated with a certain class of wavelengths, $W(c)$ affects different classes of wavelengths as the wave speed changes. 
First, we show that $W(c)$ can be optimally shaped such that the model-based streamwise energy intensity,

$$
E_{u u, W}(y)=\int_{2}^{U_{c l}} W(c) E_{u u}(y, c) \mathrm{d} c,
$$

matches the intensity of real flows at low Reynolds numbers. Then, we estimate similarity laws to approximate the optimal weight functions at high values of $\operatorname{Re}_{\tau}$. These weight functions in conjunction with the energy density of the rank-1 model with broadband forcing enable prediction of the streamwise energy intensity at technologically relevant Reynolds numbers.

\subsection{Optimal weights for small Reynolds numbers}

The weight function $W(c)$ is determined by minimizing the deviation between $E_{u u, W}(y)$ in (4.1) and the streamwise energy intensity obtained from DNS, $E_{u u, D N S}(y)$, in the interval $y^{+} \geqslant 1$ and $y \leqslant 0.8$. We do not enforce matching for $y \geqslant 0.8$ since it requires significantly large values of $W(c)$ for wave speeds close to $U_{c l}$. This is because $E_{u и}(y, c)$ is considerably smaller and more localized near the centreline compared to other locations and results in sensitivity of $W(c)$; see, for example, figure $5(a)$ for $y^{+}>24000$ corresponding to $y>0.8$ for $R e_{\tau}=30000$. Note that the main amplification mechanisms for waves with speeds close to $U_{c l}$ is the critical behaviour of the resolvent modes since the non-normality effect is small as the mean shear approaches zero. This results in small gains and resolvent modes that are localized in the wall-normal direction.

Since $E_{u u}(y, c)$ scales with $\operatorname{Re}_{\tau}^{2}$ (cf. table 3) while $E_{u u, D N S}(y)$ does not, we find the normalized weight function $\bar{W}(c)=\operatorname{Re}_{\tau}^{2} W(c)$ that solves the following optimization problem:

$$
\begin{aligned}
& \text { minimize: } \frac{\left\|E_{u u, D N S}(y)-E_{u u, W}(y)\right\|_{e}^{2}}{\left\|E_{u u, D N S}(y)\right\|_{e}^{2}}+\gamma_{w}\|\bar{W}(c)\|_{w}^{2}, \\
& \text { subject to: } \bar{W}(c)>0, \quad 2 \leqslant c \leqslant U_{c l} .
\end{aligned}
$$

Here, $\|g(y)\|_{e}$ is defined as (note integration in $\log y^{+}$)

$$
\|g(y)\|_{e}^{2}=\int_{0}^{\log \left(0.8 R e_{\tau}\right)} g^{2}\left(\log y^{+}\right) \mathrm{d} \log y^{+},
$$

to equally penalize the deviation of energy intensities near the inner peak and in the channel core. The second term in the objective function,

$$
\|\bar{W}(c)\|_{w}^{2}=\frac{1}{U_{c l}-2} \int_{2}^{U_{c l}} \bar{W}^{2}(c) \mathrm{d} c,
$$

provides the weight function with smoothness by penalizing the magnitude of $\bar{W}$, and $\gamma_{w} \geqslant 0$ controls the importance of smoothness relative to matching the model-based and DNS-based energy intensities.

The optimization problem (4.2) is solved using CVX, a package for specifying and solving convex programs in Matlab (Grant \& Boyd 2008; CVX Research, Inc 2012). We find the optimal weights for the largest Reynolds numbers that have been simulated to date using DNS, i.e. $R e_{\tau}=934$ (del Álamo et al. 2004) and $R e_{\tau}=2003$ (Hoyas \& Jiménez 2006). Even though these are orders of magnitude smaller than the Reynolds numbers for which we predict the energy intensity in $\S 4.2$, 

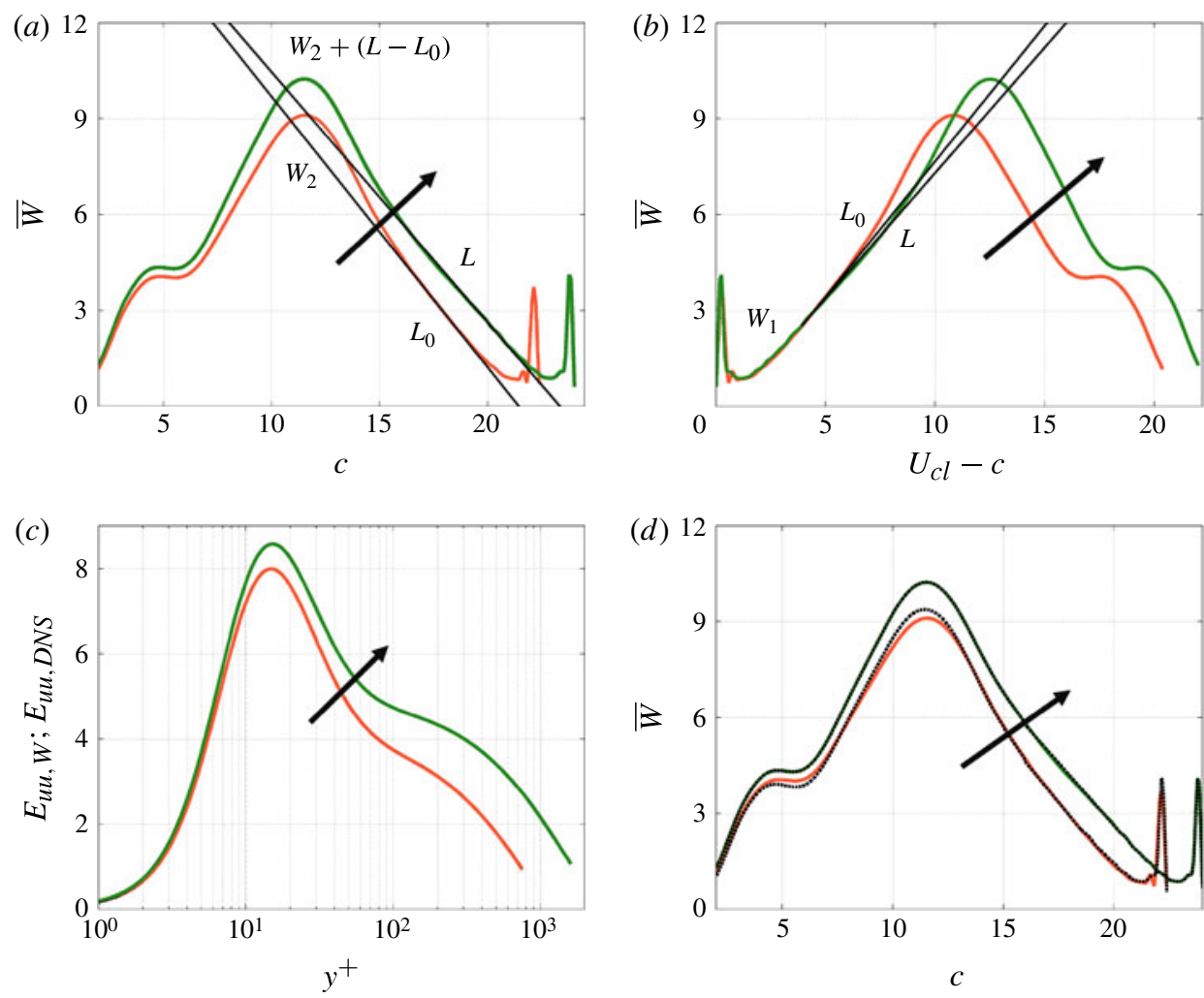

FIgURE 10. (Colour online) $(a, b)$ The optimal weight functions $\bar{W}$ as a function of $c$ in (a) and $U_{c l}-c$ in $(b)$ for $R e_{\tau}=934$ (orange) and 2003 (green). The tangent lines, $L$, to $\bar{W}\left(U_{c l}-c\right)$ at $U_{c l}-c=3.9$ for each $R e_{\tau}$ are shown in black. (c) The model-based streamwise energy intensity $E_{u u, \mathrm{~W}}\left(y^{+}\right)$for $R e_{\tau}=934$ (orange) and 2003 (green) and the DNS-based intensity $E_{u u, D N S}\left(y^{+}\right)$(black) are optimally matched by solving (4.2) for each $R e_{\tau}$. The respective curves lie on top of each other. $(d)$ The optimal weight functions $\bar{W}$ for $R e_{\tau}=934$ (orange) and 2003 (green) are compared with the weight functions obtained using the similarity law (4.6) (black dots). The arrows indicate increase in the Reynolds number.

they are free of measurement errors and useful for finding the optimal weights. We choose $\gamma_{w}=0.2$ to strike a balance between matching error $\left\|E_{u u, D N S}(y)-E_{u u, W}(y)\right\|_{e}^{2}$ and smoothness of $\bar{W}(c)$. The optimization problem is robust with respect to the choice of $\gamma_{w}$. For example, changing $\gamma_{w}$ by a factor of two has negligible effect on the matching error and the optimal weights for $7 \lesssim c \lesssim U_{c l}-2$ while slightly modifying $\bar{W}(c)$ elsewhere.

Figures $10(a)$ and $10(b)$ show the optimal weights as a function of $c$ and $U_{c l}-c$ for $R e_{\tau}=934$ and 2003. These weight functions match $E_{u u, D N S}$ and $E_{u u, W}$ with a relative error of approximately $0.2 \%$; see figure $10(c)$. As expected, $\bar{W}$ is qualitatively similar for $c \lesssim 16$ and $U_{c l}-c \lesssim 6.15$ since both the model-based and DNS-based intensities exhibit inner and outer scaling in the respective regions. Figure 10(b) shows that $\bar{W}\left(U_{c l}-c\right)$ approximately coincides for $R e_{\tau}=934$ and 2003 for $U_{c l}-c \leqslant 3.9$. We denote this universal function by $W_{1}\left(U_{c l}-c\right)$. For simplicity, the weights are approximated by linear functions in the self-similar region for $16 \leqslant c \leqslant U_{c l}-3.9$. These lines are denoted by $L$ (black) and intersect for $L(c=-2)=19.88$ and 
$L\left(U_{c l}-c=3.9\right)=2.54$. This gives an analytical expression for $L$ as a function of wave speed and Reynolds number since $U_{c l}$ varies with $R e_{\tau}$ :

$$
L\left(c ; R e_{\tau}\right)=2.54+17.34\left(\frac{U_{c l}-c-3.9}{U_{c l}-1.89}\right) .
$$

As the Reynolds number increases, $\bar{W}(c)$ is shifted upward for $c \leqslant 16$ by the kick that it receives from the self-similar region. This is expected since the DNS-based energy intensity increases with $R e_{\tau}$ in the inner region while $E_{u u}\left(y^{+}, c\right)$ remains constant. More discussion about the relationship between the weights in the self-similar and inner regions is provided in $\S 4.2$. Motivated by these observations, we formulate a similarity law for the weight function:

$$
\bar{W}\left(c ; \operatorname{Re}_{\tau}\right)= \begin{cases}W_{2}(c)+\left(L\left(c ; R e_{\tau}\right)-L_{0}(c)\right), & 2 \leqslant c \leqslant 16, \\ L\left(c ; R e_{\tau}\right), & 16<c<U_{c l}-3.9, \\ W_{1}\left(U_{c l}-c\right), & U_{c l}-3.9 \leqslant c \leqslant U_{c l},\end{cases}
$$

that consists of three segments: a universal outer segment represented by $W_{1}\left(U_{c l}-c\right)$ for $U_{c l}-3.9 \leqslant c \leqslant U_{c l}$; a Reynolds-number-dependent linear segment $L\left(c ; R e_{\tau}\right)$ that is analytically determined by (4.5); and an inner segment composed of a universal function $W_{2}(c)$ for $2 \leqslant c \leqslant 16$ superposed by a linear function $L\left(c ; R e_{\tau}\right)-L_{0}(c)$ where $L_{0}(c)=L\left(c ; R e_{\tau}=934\right)$. Figure $10(d)$ shows that the optimal weights computed by solving (4.2) are well-captured by the weights formulated using the similarity law (4.6). We note that more complex approximations could be used if more than two DNS datasets were available. Efforts to determine these weights analytically are ongoing.

\subsection{Predictions at high Reynolds numbers}

The similarity law in (4.5)-(4.6) is used to predict the weight functions, and consequently, the streamwise energy intensity at high $R e_{\tau}$ using (4.1). Figures $11(a)-11(c)$ show the predicted weights and energy intensities for $R e_{\tau}=934,2003$, 3333,10000 , and 30000. An approximately logarithmic dependence of the energy intensity on the distance from the wall is predicted at high Reynolds numbers in the logarithmic region of the mean velocity which is consistent with recent experiments (Hultmark et al. 2012; Marusic et al. 2013) and predictions of the attached-eddy hypothesis (see, for example, Perry \& Chong 1982). As evident from figure $11(d)$, the model-based predictions are consistent with the experiments of channel flows for $R e_{\tau}=3165,4000$, and 6000; especially note the comparison at $R e_{\tau}=4000$ with the data of Schultz \& Flack (2013) that maintains a sufficient spatial resolution down to the wall-normal location of the inner peak. Note that the data of Monty (2005) and the data of Schultz \& Flack (2013) at $R e_{\tau}=6000$ are not fully spatially resolved near the wall. In the absence of channel flow data at higher Reynolds numbers, figure 11(e) compares the model-based streamwise intensities with the data from boundary layer experiments for $R e_{\tau}=5813$ and 13490 (De Graaff \& Eaton 2000) and $R e_{\tau}=23013$ (Fernholz et al. 1995). Monty et al. (2009) showed that the behaviour of boundary layers, pipes, and channels is similar in the near-wall region in spite of the differences between channels/pipes and boundary layers further away from the wall. The experimental measurements are not accurate near the wall as they suffer from spatial resolution issues (see, for example, Hutchins et al. 2009). Figure $11(f)$ shows that the predicted values of the inner peak are consistent with the boundary layer measurements that are corrected (Kunkel \& Marusic 2006) based 

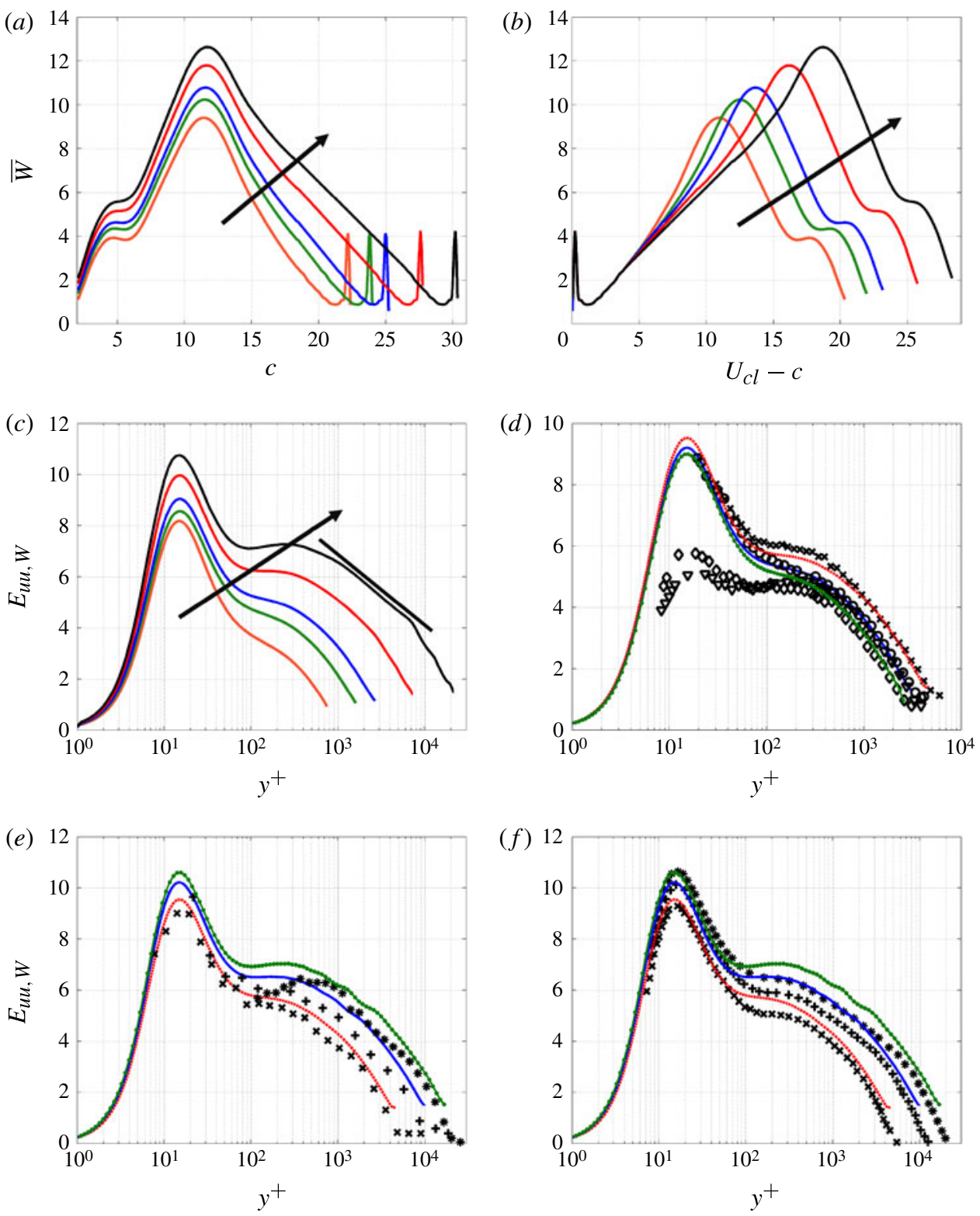

FIGURE 11. (Colour online) $(a, b)$ : The optimal weight functions $\bar{W}$ obtained using the similarity law (4.6) as a function of $c$ in $(a)$ and $U_{c l}-c$ in $(b)$. (c-f) The model-based streamwise energy intensity $E_{u u, \mathrm{~W}}\left(y^{+}\right)$for $R e_{\tau}=934$ (orange), 2003 (green), 3333 (blue), 10000 (red), and 30000 (black) in $(c) ; R e_{\tau}=3165$ (green dotted), 4000 (blue solid), and 5813 (red dashed) in $(d)$; and $R e_{\tau}=5813$ (red dashed), 13490 (blue solid), and 23013 (green dotted) in $(e, f)$. The arrows in $(a-c)$ indicate increase in the Reynolds number and the line in $(c)$ shows logarithmic scaling. The symbols in $(d)$ are experimental data from channel flows for $\operatorname{Re}_{\tau}=3165(\diamond)$ and $4000(\nabla)$ (Monty 2005), and for $R e_{\tau}=4000(\circ)$ and 6000 $(\times)$ (Schultz \& Flack 2013). The symbols in $(e)$ are experimental data from boundary layers for $R e_{\tau}=5813(\times), 13490(+)$ (De Graaff \& Eaton 2000), and $23013(\star)$ (Fernholz et al. 1995). The symbols in $(f)$ are the corrected (Kunkel \& Marusic 2006) data in the inner region of $(e)$ using the attached-eddy hypothesis. 
on the attached-eddy hypothesis. On the other hand, our predictions of the energy intensity in channel flow are larger than the data in boundary layers close to the outer peak in the middle region. This is expected since recent experiments have shown that the large structures are more energetic in internal flows such as channels and pipes compared to boundary layers (see, for example, Monty et al. 2009). This difference was attributed to the observations suggesting that the VLSMs are longer in internal flows than in boundary layers.

Obtaining the results of figure 11 requires computation of the streamwise energy density of the rank-1 model with broadband forcing at the respective values of $R e_{\tau}$. Alternatively, the universal behaviour of $E_{u u}\left(y ; \kappa_{x}, \kappa_{z}, c\right)$ can be used to avoid these computations. In the present study, we employ the universality (invariance with $\operatorname{Re}_{\tau}$ ) of $E_{\text {uu }}\left(y ; \kappa_{x}, \kappa_{z}, c\right)$ for $\mathscr{S}_{i}$ to predict the inner peak of the streamwise intensity at arbitrary high $R e_{\tau}$. Expanding the weighted energy density according to the wave speed and substituting for the weight function using the similarity law (4.6) yields

$$
\begin{aligned}
E_{u u, W}(y)= & \int_{2}^{16} \underbrace{W_{2}(c)\left(\operatorname{Re}_{\tau}^{-2} E_{u u}(y, c)\right)}_{\text {universal }}\left(1+\frac{L\left(c ; \operatorname{Re}_{\tau}\right)-L_{0}(c)}{W_{2}(c)}\right) \mathrm{d} c \\
& +\int_{16}^{U_{c l}-3.9} L\left(c ; \operatorname{Re}_{\tau}\right)\left(\operatorname{Re}_{\tau}^{-2} E_{u u}(y, c)\right) \mathrm{d} c+\int_{U_{c l}-3.9}^{U_{c l}} W_{1}(c)\left(\operatorname{Re}_{\tau}^{-2} E_{u u}(y, c)\right) \mathrm{d} c .
\end{aligned}
$$

The first integral, corresponding to the inner class of wave parameters $\mathscr{S}_{i}$, contains a universal function multiplied by a coefficient $L\left(c ; R e_{\tau}\right)$ that also appears in the second integral for the faster and larger waves in the self-similar region. It represents the contribution from the inner class of wave parameters that are coupled with and amplified by the large scales in the self-similar region. This is similar to the model that Marusic et al. (2010b) proposed to capture the influence of the large scales $u_{L}$ (close to the geometric mean of the middle region of $U$ ) on the small scales $u_{S}$ close to the inner peak of the energy intensity:

$$
u_{S}=u^{*}\left(1+\beta u_{L}\right)+\alpha u_{L} .
$$

For the purpose of the present study, (4.8) implies that the small structures are determined by a universal inner-scaled function $u^{*}$ multiplied by a coefficient $1+\beta u_{L}$ that increases with the energy of the large structures. Physically, the first term in (4.8) describes the amplitude modulation of small scales by the large scales and the second term represents the direct superimposition of the large scales on the inner-scaled near-wall peak (Marusic et al. 2010b).

The blue long-dashed curve in figure 12(a) shows the contribution of the universal function in (4.7) to the energy intensity. This is equal to the contribution of the inner class of wave parameters to the energy intensity for $R e_{\tau}=934$, i.e. for $L=L_{0}$. In other words, the inner class of wave parameters is not influenced by the large scales in the middle region for $R e_{\tau}=934$. This is expected since at $R e_{\tau} \approx 1000$, the inner and outer scales are separated, in the (temporal) frequency domain, by the wave speed $c=16$ : i.e. inner scales for $c<16$ and outer scales for $c>U_{c l}-6.15 \approx 16$. Therefore, $R e_{\tau} \approx 1000$ is the smallest Reynolds number where the purely inner and outer scales are separated in the wavenumber-frequency domain. Notice that the abovementioned scale separation in the frequency domain does not contradict the weak scale separation in the premultiplied spectra at $R e_{\tau} \approx 1000$. The latter is a consequence of 
(a)

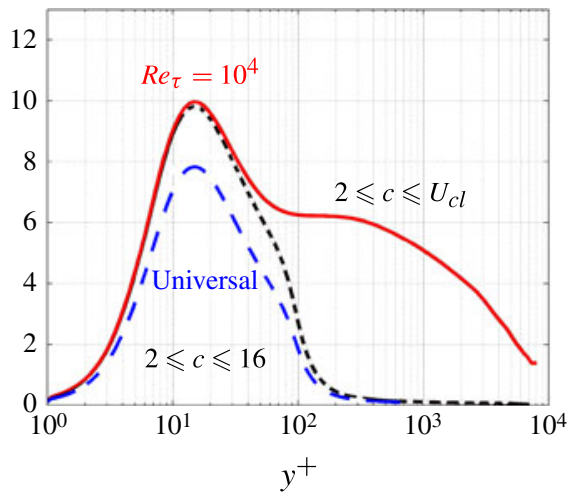

(b)

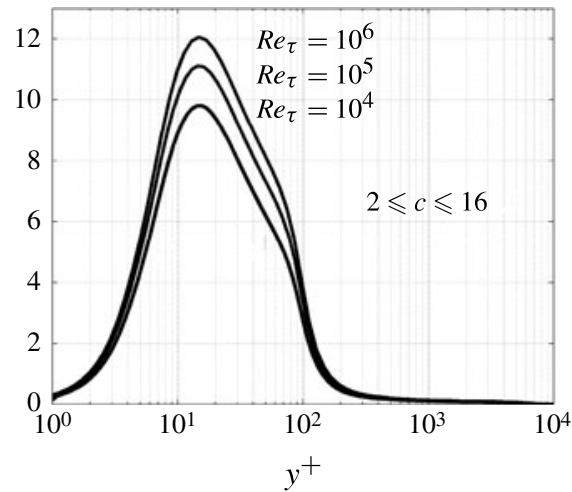

FIgURE 12. (Colour online) (a) The red solid curve is the model-based energy intensity $E_{u u, W}(y)$ for $\operatorname{Re}_{\tau}=10^{4}$. The blue long-dashed curve is the contribution from the universal function in (4.7), and the black short-dashed curve is the contribution from the inner class of wave parameters $\mathscr{S}_{i}$. (b) The contribution to the energy intensity from the inner class of wave parameters for $R e_{\tau}=10^{4}, 10^{5}$, and $10^{6}$.

time-averaging that overlays the separated scales in the frequency domain such that the distinction of different scales in the spatial spectra becomes difficult.

The black short-dashed curve shows the contribution of the first integral in (4.7) to the streamwise intensity for $\operatorname{Re}_{\tau}=10^{4}$. Notice that the large scales from the selfsimilar region increase the inner peak by amplifying the universal function through the coefficient $1+\left(L-L_{0}\right) / W_{2}$. The red solid curve is the total intensity obtained by integrating the contribution of all wave parameters $\mathscr{S}_{e}$. The inner peak is captured by the first integral and the direct superimposition of the large scales on the inner peak is negligible. Therefore, the first integral readily yields the behaviour of the streamwise intensity near the inner peak. For example, figure $12(b)$ illustrates how the more energetic large scales at $R e_{\tau}=10^{5}$ and $10^{6}$ further increase the inner peak relative to $R e_{\tau}=10^{4}$ by amplifying the universal function $W_{2}$.

Figure 13 is adapted from figure 8 in Marusic et al. (2010a) where the DNS and experimental data from channels and boundary layers are summarized (open and filled black symbols). The black filled squares and circles, respectively, show the magnitude of the inner $\left(y^{+}=15\right)$ and outer $\left(y^{+}=3.9 R e_{\tau}^{1 / 2}\right)$ peaks in recent boundary layer experiments (Marusic et al. 2010a). Using these data, the authors proposed two possibilities for the behaviour of the inner peak at high Reynolds numbers. The first possibility is to extrapolate following the trend suggested by the filled black squares (line 1). The second possibility, motivated by the fact that the large scales increase the energy of the small scales, is to extrapolate following line 3 which is parallel to line 2 that captures the variation of the outer peak with $R e_{\tau}$. The data (open triangles) from large-eddy simulations of boundary layers (Inoue et al. 2012) combined with the wall model of Marusic et al. (2010b) are shown for comparison. The current understanding, at least for relatively small intervals of Reynolds numbers, suggests logarithmic growth of the inner peak. However, due to lack of sufficient spatial resolution close to the wall, the available experimental data obtained for different ranges of $R e_{\tau}$, predict different rates for the logarithmic growth, e.g. see Marusic et al. (2010a). Therefore, the available data are not sufficient for predicting the exact behaviour of the inner peak as $R e_{\tau}$ increases. 


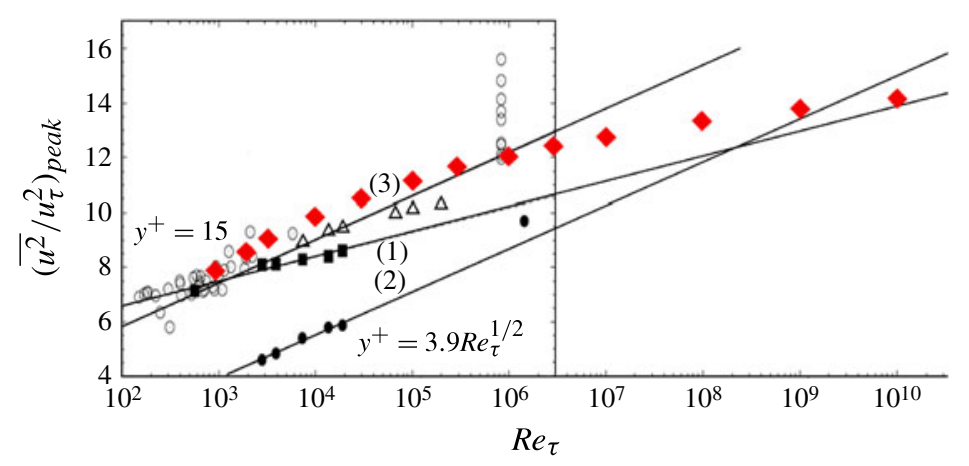

FIGURE 13. (Colour online) Variation of the inner $\left(y^{+}=15\right)$ and outer $\left(y^{+}=3.9 R e_{\tau}^{1 / 2}\right)$ peaks of the streamwise energy intensity with Reynolds number. The figure is adapted from Marusic et al. (2010a). The black open and filled symbols are experimental and simulation data from channels and boundary layers, see Hutchins \& Marusic (2007a) for a full list of references. The open triangles are from large-eddy simulations of boundary layers (Inoue et al. 2012). The diamonds are the predicted inner peak intensities obtained from the present model for turbulent channels. The lines show linear extrapolation of data at high Reynolds numbers.

The diamonds in figure 13 show the model-based prediction of the inner peak of the streamwise intensity up to $R e_{\tau}=10^{10}$. These predictions are made at no additional cost using the universal energy density for the inner class of wave parameters and the similarity law for the weight functions. These results are obtained for channels and are potentially different than boundary layers. In spite of an approximately logarithmic growth of the predicted inner peak up to $R e_{\tau} \sim 10^{6}$, a sub-logarithmic behaviour becomes evident when seven decades of $R e_{\tau}$ are considered. As shown in (4.7), the linear part of the weight function, modelling the influence of large outer-scaled modes on the small inner-scaled modes, affects the growth of the inner peak with $R e_{\tau}$. The sub-logarithmic growth of the predicted inner peak can be attributed to the decrease in the slope of $L(c)$ as $R e_{\tau}$ increases, cf. (4.5) and figure 10(a). Understanding the Reynolds-number dependence of $L(c)$ is an essential part of our ongoing research which is focused on analysis of the self-similar modes in the logarithmic region.

\section{Concluding remarks}

Starting from the NSE, we highlighted the low-rank nature of the resolvent, formulated for individual wall-parallel wavenumbers and frequencies, and illustrated its power by showing that the most energetic motions of real turbulent flows correspond to wavenumbers and frequencies whose resolvent is approximately rank-1 (in the wallnormal direction). Motivated by this observation, we studied the streamwise energy density of the rank-1 model subject to forcings in the wall-parallel directions and time that were broadband and optimized, or 'trained', with respect to the available DNS data.

Our analysis consists of two steps: first, identifying the modes that are highly amplified by the NSE and their scaling (essentially an analysis of the resolvent operator), and then calculating weighting functions (by matching to DNS results) which determine which of these modes will be sustained in the real flow (connecting the linear system of resolvent operators back to the full NSE). 


\subsection{Scaling of the most amplified resolvent modes}

It was shown that the resolvent admits three classes of wavenumbers and wave speeds for which the corresponding principal singular values and singular functions exhibit universal behaviour with Reynolds number. These classes are directly related to the universal regions of the turbulent mean velocity (which is assumed known a priori) and thus are primarily distinguished by the wave speed: (i) a truly inner-scaled class of waves with constant speeds in the inner region of the turbulent mean velocity; (ii) a class of waves with outer-scaled height and width and constant defect speeds relative to the centreline; and (iii) a class of waves with outer-scaled length and constant defect speeds relative to the geometric mean of the middle region of the turbulent mean velocity. In addition, we showed that hierarchies of geometrically self-similar modes whose length and width respectively scale quadratically and linearly with their height are admitted by the resolvent in the presence of a logarithmic mean velocity.

The integral role of wave speed and critical layers in characterizing the classes of universal modes with Reynolds number and the geometrically self-similar modes with the wall-normal distance is understood and emphasized for the first time. The conventional understanding about the scales of turbulent flows comes from the timeaveraged velocity spectra in DNS and experiments. Upon integration in time, the separated scales in the (temporal) frequency domain are overlaid, and distinction of different scales in the spatial spectra becomes difficult. Therefore, the identified scales have significant implications for understanding the scaling of wall turbulence. They are inherent features of the linear mechanisms in the NSE and, consequently, the energy extraction mechanisms from the mean velocity. In both the universal and self-similar classes, the wall-normal length scale is inherited from the turbulent mean velocity, and the wall-parallel length scales are determined from the balance between the viscous dissipation term, $\left(1 / R e_{\tau}\right) \Delta$, and the mean advection terms in the resolvent, e.g. i $\kappa_{x}(U-c)$.

The main results of the present paper, i.e. the scalings identified in $\S 3$, rely on the accepted scales of the turbulent mean velocity and, otherwise, do not depend on the exact shape of $U$. Therefore, the choice of eddy viscosity or the von Kármán constant $\kappa$ does not change our main results. On the other hand, the debate on the universality and/or exact value of $\kappa$ is ongoing, e.g. see Nagib \& Chauhan (2008), and using the turbulent viscosity given in (2.3) can result in inaccuracies in the considered mean velocity. This can affect the quantitative results of $\S 3$ and $\S 4$, e.g. the shape of the resolvent modes and the predicted growth rate of the inner peak. Characterizing these effects is a topic of future work, and the sensitivity is known to be highest in the region of highest shear, close to the wall. Since closing the feedback loop in figure 2 eventually generates the exact turbulent mean velocity, we do not over-emphasize the quantitative results of the present study.

We highlight the uniqueness of the identified scales, meaning that there are no other scales that result in universal or geometrically self-similar principal resolvent modes. In addition, the difference between the scalings of the resolvent modes and real turbulent flows implies the need for distinguishing the resolvent modes from the weighted modes that represent the real turbulent flow. For example, the scaling admitted by the self-similar resolvent modes yields $\lambda_{z}^{3} \sim \lambda_{x} y_{c}$ which is different from the trend $\lambda_{z}^{2} \sim \lambda_{x} y$ observed in the DNS-based two-dimensional streamwise spectrum (Jiménez \& Hoyas 2008). Understanding the scaling differences between the resolvent modes and the weighted modes requires detailed scrutiny of the weights and the nonlinear effects, a topic of ongoing research. In addition, our results suggest 
that, owing to scale separation in frequency, there is a large benefit to obtaining and analysing the scaling of three-dimensional time-resolved spectral measurements.

\subsection{Effect of nonlinearity}

From a systems theory point of view, the nonlinear terms wrap a feedback loop around the linear sub-systems in the NSE and redistribute the energy. They determine the wall-normal shape and the magnitude/phase of the driving force for an individual mode. Therefore, the real flow is obtained by superposing the resolvent response modes that are weighted according to projection of the driving force on the resolvent forcing modes.

We started by assuming that the nonlinear forcing is broadband in the wall-parallel directions and time and aligned in the principal resolvent forcing modes. It was shown that these simple assumptions can qualitatively produce different scaling regions of the streamwise energy spectra. Therefore, the proposed analysis effectively narrows down the scaling problem in wall-bounded turbulent flows to the problem of understanding the influence of nonlinearity on the inevitable scales that are admitted by the linear mechanisms, i.e. determining which of those admitted modes will be required in real flows for the flow to be self-sustaining. A full description of the latter effects is beyond the scope of the present paper, but the subject of ongoing work.

A non-broadband forcing in time was accounted for by considering a weight function in the wave speed. We showed that 'training' the weights based on the wave speed can result in streamwise energy intensities that quantitatively match DNS and experiments. As the Reynolds number increases, the optimal weight functions increase for wave speeds in the inner region of the mean velocity. Representation of the optimal weights using similarity laws revealed that the amount of upward shift is linearly correlated with the weight function for wave speeds in the middle region of the mean velocity. In other words, the weight function increases with the energy intensity of the large scales and amplifies the universal inner-scaled energy density of the rank-1 model. Therefore, it implicitly captures the well-known coupling of small scales with the large scales and their subsequent amplification in real turbulent flows.

A consequence of the simplicity of the identified scaling in the wavenumber-frequency domain is the success of the simple weighting based on convection velocity in post- and pre-dicting the variation of the streamwise velocity fluctuations with Reynolds number. One of the main results of this study is that the rank-1 approximation, together with the optimal weight functions and the (well-known) mean velocity profile, is sufficient for predicting the streamwise energy intensity at high Reynolds numbers. Even though the weight function provides a rough intuition about the effect of nonlinearity, the explicit analysis of the nonlinear feedback on the velocity field remains a subject of ongoing research.

\subsection{Outlook of the present analysis as a predictive tool}

The present study effectively divides the streamwise energy density of the rank-1 model with broadband forcing into inner- and outer-scaled universal regions with Reynolds number and a geometrically self-similar region with distance from the wall that bridges the gap between the inner and outer regions. This enables scaling of the streamwise energy density to arbitrary large Reynolds numbers. It was shown that the most energetic wave parameters and the corresponding scales roughly agree with the dominant near-wall motions in real turbulent flows.

The identified self-similar resolvent modes facilitate analytical developments in the logarithmic region of the turbulent mean velocity and can result in significant 
simplifications in analysis of wall turbulence. In addition, the wall-normal locality of the self-similar modes in a given hierarchy suggests that the linear sub-systems in the NSE impose a direct correspondence between wall-parallel scales and wall-normal locations in the logarithmic region. In the classical cascade analogy, e.g. see the review paper by Jiménez (2012), this is reminiscent of an inertial regime, the study of which is a topic of ongoing research. Furthermore, ongoing research is focused on utilizing the identified scalings to better understand the structure and evolution of the hypothesized attached eddies.

The available predictive models of wall turbulence, e.g. the attached-eddy hypothesis (Townsend 1976; Perry \& Chong 1982) and the model of Marusic \& Kunkel (2003), rely on physical intuition that is gained from DNS and experiments. For example, the method proposed by Marusic \& Kunkel (2003) is based on an assumption about the influence of outer-layer modes on the near-wall modes (their equation (2)), where the underlying functions are determined by empirical curve fits to the experimental data (their equations (3)-(5)). The present model is more fundamental as it directly uses the NSE for decomposing the flow into classes of modes that are uniquely scaled with the Reynolds number and distance from the wall. Since the wall-normal shape of these modes is one of the model outputs, the contribution of the present work goes beyond reporting an empirical fit to the model-based data, namely by exploring the scaling of the modes admitted by the NSE.

In essence, this work supports the efficacy of the low-rank model of wall turbulence proposed by McKeon \& Sharma (2010) by demonstrating that it can be used both to determine self-similar mode scalings and to obtain a low-rank representation of the streamwise intensity, given appropriate, self-similar weighting of the modes. Our ongoing research, to be reported elsewhere, is focused on analytical expression of the streamwise energy density for wave speeds in the logarithmic region of the mean velocity and a priori derivation of the weight functions. Addressing the limitations and implications of the low-rank model for predicting the wall-normal and spanwise energy spectra as well as the Reynolds stresses is another topic of future research.

\section{Acknowledgements}

The support of Air Force Office of Scientific Research under grants FA 9550-091-0701 (P. M. J. Schmisseur) and FA 9550-12-1-0469 (P. M. D. Smith) is gratefully acknowledged.

\section{Appendix A. Derivation of the inner scalings}

We show that the transfer function $H$ admits universal behaviour for the modes with speeds $c \lesssim 16$. For these modes, following (3.5), the $y$-dependent coefficients in the transfer function $H$, are either independent of $R e_{\tau}$, e.g. $U\left(y^{+}\right)-c$, or scale with $R e_{\tau}$, e.g. $U^{\prime}\left(y^{+}\right)$. This allows scaling the height of the resolvent modes with the viscous unit $\nu / u_{\tau}$. In addition, the balance between the viscous dissipation term, $\left(1 / R_{\tau}\right) \Delta$, and the mean advection terms, e.g. i $\kappa_{x}(U-c)$, in the resolvent in (2.9) requires scaling of the wall-parallel wavelengths with the viscous unit $v / u_{\tau}$ :

$$
\lambda_{x}^{+}=R e_{\tau} \lambda_{x}, \quad y^{+}=R e_{\tau} y, \quad \lambda_{z}^{+}=R e_{\tau} \lambda_{z} .
$$

The differential operators in $y$ and the wavenumber symbols in the inner coordinates are

$$
\partial / \partial_{y^{+}}=R e_{\tau}^{-1} \partial / \partial_{y}, \quad \kappa_{x}^{+}=R e_{\tau}^{-1} \kappa_{x}, \quad \kappa_{z}^{+}=R e_{\tau}^{-1} \kappa_{z}, \quad \Delta^{+}=R e_{\tau}^{-2} \Delta .
$$


Consequently in the inner coordinates, the operators $R_{A}, C$, and $C^{\dagger}$ in (2.7) and (2.9) scale as

$$
\begin{aligned}
R_{A} & =\left[\begin{array}{cc}
R e_{\tau} X_{1} & 0 \\
R e_{\tau}^{2} X_{3} & R e_{\tau} X_{2}
\end{array}\right]^{-1}=\left[\begin{array}{cc}
\operatorname{Re}_{\tau}^{-1} Y_{1} & 0 \\
Y_{3} & R e_{\tau}^{-1} Y_{2}
\end{array}\right], \\
C & =\left[\begin{array}{cc}
C_{1} & R e_{\tau}^{-1} C_{2} \\
C_{3} & 0 \\
C_{4} & R e_{\tau}^{-1} C_{5}
\end{array}\right], \quad C^{\dagger}=\left[\begin{array}{ccc}
C_{1}^{\dagger} & C_{3}^{\dagger} & C_{4}^{\dagger} \\
R e_{\tau} C_{2}^{\dagger} & 0 & R e_{\tau} C_{5}^{\dagger}
\end{array}\right] .
\end{aligned}
$$

For given $\kappa_{x}^{+}$and $\kappa_{z}^{+}$, the operators $C_{1}$ to $C_{5}$ and their adjoints are independent of $R e_{\tau}$. On the other hand, the operators $X_{1}$ to $X_{3}$ and $Y_{1}$ to $Y_{3}$ contain spatially varying coefficients, $U-c$ and its first two derivatives, that depend on $R e_{\tau}$. As discussed at the beginning of $\S 3, U$ scales with $y^{+}$and is independent of $R e_{\tau}$ for $y^{+} \lesssim 100$. Therefore, for given $\kappa_{x}^{+}, \kappa_{z}^{+}$, and $c \lesssim U\left(y^{+}=100\right)=16$, the operators $X_{1}$ to $X_{3}$ and $Y_{1}$ to $Y_{3}$ are independent of $R e_{\tau}$ when acting on functions whose supports are inside the interval $0<y^{+} \lesssim 100$. Since the principal resolvent modes are localized around the critical layer (i.e. the wall-normal location where the turbulent mean velocity equals $c$ ), the resolvent modes are negligible outside $y^{+} \lesssim 100$ for $c \lesssim 16$ and all of the aforementioned operators are effectively independent of $R e_{\tau}$. It follows from (A 1) that

$$
H=C R_{A} C^{\dagger}=\left[\begin{array}{lll}
R e_{\tau}^{-1} H_{11} & R e_{\tau}^{-1} H_{12} & R e_{\tau}^{-1} H_{13} \\
R e_{\tau}^{-1} H_{21} & R e_{\tau}^{-1} H_{22} & R e_{\tau}^{-1} H_{23} \\
R e_{\tau}^{-1} H_{31} & R e_{\tau}^{-1} H_{32} & R e_{\tau}^{-1} H_{33}
\end{array}\right],
$$

where the operators $H_{i j}$ are effectively independent of $R e_{\tau}$ when acting on their principal resolvent modes. Therefore, the principal singular value of $H$ is proportional to $R e_{\tau}^{-1}$. In addition, the orthonormality constraints (2.11) on $\hat{\psi}_{1}$ and $\hat{\boldsymbol{\phi}}_{1}$ require that these functions scale as $R e_{\tau}^{1 / 2}$. This is because the supports of $\hat{\psi}_{1}$ and $\hat{\boldsymbol{\phi}}_{1}$ are independent of $R e_{\tau}$ in inner units (hence, proportional to $R e_{\tau}^{-1}$ in outer units). In other words, $\hat{\boldsymbol{\psi}}_{1}(y)$ and $\hat{\boldsymbol{\phi}}_{1}(y)$ become thinner and taller as $R e_{\tau}$ increases. Finally, the streamwise energy density $E_{u u}=\kappa_{x}^{2} \kappa_{z} \sigma_{1}^{2}\left|u_{1}\right|^{2}$ scales with

$$
\left(\operatorname{Re}_{\tau}\right)^{2}\left(\operatorname{Re}_{\tau}\right)\left(\operatorname{Re}_{\tau}^{-1}\right)^{2}\left(\operatorname{Re}_{\tau}^{1 / 2}\right)^{2}=\operatorname{Re}_{\tau}^{2} .
$$

\section{Appendix B. Derivation of the outer scalings}

For the modes with defect speeds $0 \lesssim U_{c l}-c \lesssim 6.15$, we show that the transfer function $H$ admits universal behaviour with Reynolds number. For these modes, following (3.5), the $y$-dependent coefficients in the transfer function $H$, e.g. $U(y)-c$, are independent of $R e_{\tau}$. This allows scaling the height of the resolvent modes with $h$. Furthermore, the balance between the viscous dissipation term, $\left(1 / R_{\tau}\right) \Delta$, and the mean advection terms, e.g. i $\kappa_{x}(U-c)$, in the resolvent in (2.9) requires scaling of the spanwise coordinate with $h$ and the streamwise coordinate with $h R e_{\tau}$. Therefore, the streamwise wavenumber symbol in the outer coordinates is given by $\kappa_{x}^{-}=\operatorname{Re}_{\tau} \kappa_{x}$. The Laplacian

$$
\Delta=\partial_{y y}-R e_{\tau}^{-2}\left(\kappa_{x}^{-}\right)^{2}-\kappa_{z}^{2},
$$


is independent of $R e_{\tau}$ if $\kappa_{z}^{2}$ dominates $R e_{\tau}^{-2}\left(\kappa_{x}^{-}\right)^{2}$ for all values of $R e_{\tau}$. For fixed $\kappa_{x}^{-}$ and $\kappa_{z}$, it suffices that

$$
\frac{\lambda_{x}^{-}}{\lambda_{z}}=\frac{\kappa_{z}}{\kappa_{x}^{-}} \gtrsim \frac{\gamma}{R e_{\tau, \min }} .
$$

In the outer coordinates, the operators $R_{A}, C$, and $C^{\dagger}$ in (2.7) and (2.9) scale as

$$
\begin{aligned}
R_{A} \approx & {\left[\begin{array}{cc}
\operatorname{Re}_{\tau}^{-1} \tilde{X}_{1} & 0 \\
\tilde{X}_{3} & R e_{\tau}^{-1} \tilde{X}_{2}
\end{array}\right]^{-1}=\left[\begin{array}{cc}
R e_{\tau} \tilde{Y}_{1} & 0 \\
\operatorname{Re}_{\tau}^{2} \tilde{Y}_{3} & R e_{\tau} \tilde{Y}_{2}
\end{array}\right], } \\
C & \approx\left[\begin{array}{cc}
\operatorname{Re}_{\tau}^{-1} \tilde{C}_{1} & \tilde{C}_{2} \\
\tilde{C}_{3} & 0 \\
\tilde{C}_{4} & R e_{\tau}^{-1} \tilde{C}_{5}
\end{array}\right], \quad C^{\dagger} \approx\left[\begin{array}{ccc}
R e_{\tau}^{-1} \tilde{C}_{1}^{\dagger} & \tilde{C}_{3}^{\dagger} & \tilde{C}_{4}^{\dagger} \\
\tilde{C}_{2}^{\dagger} & 0 & R e_{\tau}^{-1} \tilde{C}_{5}^{\dagger}
\end{array}\right] .
\end{aligned}
$$

For given $\kappa_{x}^{-}$and $\kappa_{z}$ that satisfy the constraint (B 2), the operators $\tilde{C}_{1}$ to $\tilde{C}_{5}$ and their adjoints are approximately independent of $\operatorname{Re}_{\tau}$. In addition, the defect velocity $U_{c l}-U(y)$ is independent of $\operatorname{Re}_{\tau}$ for $y \gtrsim 0.1$. Therefore, for given $\kappa_{x}^{-}, \kappa_{z}$, and $U_{c l}-c \lesssim U_{c l}-U(y=0.1)=6.15$, the operators $\tilde{X}_{1}$ to $\tilde{X}_{3}$ and $\tilde{Y}_{1}$ to $\tilde{Y}_{3}$ are approximately independent of $\operatorname{Re}_{\tau}$ when acting on functions whose supports are inside the interval $0.1 \lesssim y \lesssim 1$. From (B 2), we have

$$
H=C R_{A} C^{\dagger} \approx\left[\begin{array}{ccc}
\operatorname{Re}_{\tau} \tilde{H}_{11} & \operatorname{Re}_{\tau}^{2} \tilde{H}_{12} & \operatorname{Re}_{\tau}^{2} \tilde{H}_{13} \\
\tilde{H}_{21} & \operatorname{Re}_{\tau} \tilde{H}_{22} & \operatorname{Re}_{\tau} \tilde{H}_{23} \\
\tilde{H}_{31} & \operatorname{Re}_{\tau} \tilde{H}_{32} & \operatorname{Re}_{\tau} \tilde{H}_{33}
\end{array}\right] .
$$

Owing to the locality of the principal resolvent modes around the critical layer, the operators $\tilde{H}_{i j}$ are approximately independent of $\operatorname{Re}_{\tau}$ when acting on their principal resolvent modes. Therefore, the principal singular value of $H$ is proportional to $R e_{\tau}^{2}$. Since $\hat{\boldsymbol{\psi}}_{1}$ and $\hat{\boldsymbol{\phi}}_{1}$ scale in the outer length scale, the orthonormality constraints (2.11) require that these functions be independent of $R e_{\tau}$. Finally, the streamwise energy density $E_{u u}=\kappa_{x}^{2} \kappa_{z} \sigma_{1}^{2}\left|u_{1}\right|^{2}$ scales with

$$
\left(\operatorname{Re}_{\tau}^{-1}\right)^{2}(1)\left(\operatorname{Re}_{\tau}^{2}\right)^{2}(1)^{2}=\operatorname{Re}_{\tau}^{2}
$$

\section{Appendix C. Derivation of the geometrically self-similar scalings}

The transfer function $H$ admits geometrically self-similar modes with speeds in the logarithmic region of the turbulent mean velocity. In this region, it follows from the discussion in $\S 3.2$ that the $y$-dependent coefficient in the transfer function $H$ can be expressed as $U(y)-c=(1 / \kappa) \log \left(y / y_{c}\right)$, where $y_{c}$ is the critical wall-normal location corresponding to $c$, i.e. $c=U\left(y_{c}\right)$. Similarly, $U^{\prime}$ and $U^{\prime \prime}$ are functions of $y / y_{c}$. This allows scaling the height of the resolvent modes with $y_{c}$. Furthermore, the balance between the viscous dissipation term, $\left(1 / R_{\tau}\right) \Delta$, and the mean advection terms, e.g. $\mathrm{i} \kappa_{x}(U-c)$, in the resolvent in (2.9) requires scaling of the spanwise wavelength with $y_{c}$ and the streamwise wavelength with $y_{c}^{+} y_{c}$,

$$
\bar{\lambda}_{x}=\lambda_{x} /\left(y_{c}^{+} y_{c}\right), \quad \bar{y}=y / y_{c}, \quad \bar{\lambda}_{z}=\lambda_{z} / y_{c} .
$$


The differential operators in $y$ and the wavenumber symbols in the $y_{c}$-scaled coordinates are

$$
\partial / \partial_{\bar{y}}=y_{c}\left(\partial / \partial_{y}\right), \quad \bar{\kappa}_{x}=\left(y_{c}^{+} y_{c}\right) \kappa_{x}, \quad \bar{\kappa}_{z}=y_{c} \kappa_{z} .
$$

For given $\bar{\kappa}_{x}$ and $\bar{\kappa}_{z}$, the Laplacian

$$
\Delta=y_{c}^{-2}\left(\partial_{\bar{y} \bar{y}}-\left(y_{c}^{+}\right)^{-2}\left(\bar{\kappa}_{x}\right)^{2}-\left(\bar{\kappa}_{z}\right)^{2}\right),
$$

approximately scales with $y_{c}^{-2}$ if $\left(\bar{\kappa}_{z}\right)^{2}$ dominates $\left(y_{c}^{+}\right)^{-2}\left(\bar{\kappa}_{x}\right)^{2}$, i.e.

$$
\kappa_{z} / \kappa_{x}=\lambda_{x} / \lambda_{z}=y_{c}^{+}\left(\bar{\lambda}_{x} / \bar{\lambda}_{z}\right) \gtrsim \gamma,
$$

where a conservative value for $\gamma$ is $\sqrt{10}$. Since the aspect ratio $\lambda_{x} / \lambda_{z}$ increases with $y_{c}^{+}$, the smallest value of $y_{c}^{+}$for which (C4) is guaranteed is equal to $y_{c_{1}}^{+}=\gamma\left(\bar{\lambda}_{z} / \bar{\lambda}_{x}\right)$. Therefore, the smallest wave speed that satisfies the aspect-ratio constraint and lies above the inner region is given by

$$
c_{1}=\max \left(16, B+(1 / \kappa) \log y_{c_{1}}^{+}\right) .
$$

Then, the operators $R_{A}, C$, and $C^{\dagger}$ in (2.7) and (2.9) scale as

$$
\begin{aligned}
R_{A} & =\left[\begin{array}{cc}
\left(y_{c}^{+} y_{c}\right)^{-1} \bar{X}_{1} & 0 \\
y_{c}^{-2} \bar{X}_{3} & \left(y_{c}^{+} y_{c}\right)^{-1} \bar{X}_{2}
\end{array}\right]^{-1}=\left[\begin{array}{cc}
\left(y_{c}^{+} y_{c}\right) \bar{Y}_{1} & 0 \\
\left(y_{c}^{+}\right)^{2} \bar{Y}_{3} & \left(y_{c}^{+} y_{c}\right) \bar{Y}_{2}
\end{array}\right], \\
C & =\left[\begin{array}{cc}
\left(1 / y_{c}^{+}\right) \bar{C}_{1} & \left(y_{c}\right) \bar{C}_{2} \\
\bar{C}_{3} & 0 \\
\bar{C}_{4} & \left(1 / R e_{\tau}\right) \bar{C}_{5}
\end{array}\right], \quad C^{\dagger}=\left[\begin{array}{ccc}
\left(1 / y_{c}^{+}\right) \bar{C}_{1}^{\dagger} & \bar{C}_{3}^{\dagger} & \bar{C}_{4}^{\dagger} \\
\left(1 / y_{c}\right) \bar{C}_{2}^{\dagger} & 0 & \left(y_{c}^{+} y_{c}\right)^{-1} \bar{C}_{5}^{\dagger}
\end{array}\right] .
\end{aligned}
$$

For given $\bar{\kappa}_{x}$ and $\bar{\kappa}_{z}$ that satisfy the constraint (C4), the operators $\bar{C}_{1}$ to $\bar{C}_{5}$ and their adjoints are approximately independent of $y_{c}$ and $R e_{\tau}$. In addition, the operators $\bar{X}_{1}$ to $\bar{X}_{3}$ and $\bar{Y}_{1}$ to $\bar{Y}_{3}$ are approximately independent of $y_{c}$ and $R e_{\tau}$ when acting on functions whose supports are localized in the interval $100 / R e_{\tau} \leqslant y \leqslant 0.1$. From (C 3$)$, we have

$$
H=C R_{A} C^{\dagger}=\left[\begin{array}{ccc}
\left(y_{c}^{+} y_{c}\right) \bar{H}_{11} & \left(y_{c}^{+}\right)^{2}\left(y_{c}\right) \bar{H}_{12} & \left(y_{c}^{+}\right)^{2}\left(y_{c}\right) \bar{H}_{13} \\
\left(y_{c}\right) \bar{H}_{21} & \left(y_{c}^{+} y_{c}\right) \bar{H}_{22} & \left(y_{c}^{+} y_{c}\right) \bar{H}_{23} \\
\left(y_{c}\right) \bar{H}_{31} & \left(y_{c}^{+} y_{c}\right) \bar{H}_{32} & \left(y_{c}^{+} y_{c}\right) \bar{H}_{33}
\end{array}\right],
$$

where the operators $\bar{H}_{i j}$ are effectively independent of $y_{c}$ and $R e_{\tau}$ when acting on their principal resolvent modes. Therefore, the principal singular value of $H$ is proportional to $\left(y_{c}^{+}\right)^{2}\left(y_{c}\right)$. In addition, the orthonormality constraints (2.11) on $\hat{\psi}_{1}$ and $\hat{\boldsymbol{\phi}}_{1}$ require that these functions scale with $\left(y_{c}\right)^{-1 / 2}$. This is because the supports of $\hat{\boldsymbol{\psi}}_{1}$ and $\hat{\boldsymbol{\phi}}_{1}$ expand with $y_{c}$. Finally, the streamwise energy density $E_{u u}=\kappa_{x}^{2} \kappa_{z} \sigma_{1}^{2}\left|u_{1}\right|^{2}$ for the waves that belong to the same hierarchy scales with

$$
\left(y_{c}^{+} y_{c}\right)^{-2}\left(y_{c}\right)^{-1}\left(\left(y_{c}^{+}\right)^{2}\left(y_{c}\right)\right)^{2}\left(y_{c}\right)^{-1}=R e_{\tau}^{2} .
$$

\section{REFERENCES}

Adrian, R. J. 2007 Hairpin vortex organization in wall turbulence. Phys. Fluids 19, 041301.

Adrian, R. J., Meinhart, C. D. \& Tomkins, C. D. 2000 Vortex organization in the outer region of the turbulent boundary layer. J. Fluid Mech. 422, 1-54. 
AfZAL, N. 1984 Mesolayer theory for turbulent flows. AIAA J. 22, 437-439.

DEL Álamo, J. C. \& JimÉNEZ, J. 2006 Linear energy amplification in turbulent channels. J. Fluid Mech. 559, 205-213.

Del Álamo, J. C. \& JimÉnez, J. 2009 Estimation of turbulent convection velocities and corrections to Taylor's approximation. J. Fluid Mech. 640, 5-26.

del Álamo, J. C., Jiménez, J., Zandonade, P. \& Moser, R. D. 2004 Scaling of the energy spectra of turbulent channels. J. Fluid Mech. 500, 135-144.

del Álamo, J. C., Jiménez, J., Zandonade, P. \& Moser, R. D. 2006 Self-similar vortex clusters in the turbulent logarithmic region. J. Fluid Mech. 561, 329-358.

Alfredsson, P. H., Örlü, R. \& Segalini, A. 2012 A new formulation for the streamwise turbulence intensity distribution in wall-bounded turbulent flows. Eur. J. Mech. (B/Fluids) 36, $167-175$.

Bailey, S. C. C., Hultmark, M., Smits, A. J. \& Schultz, M. P. 2008 Azimuthal structure of turbulence in high Reynolds number pipe flow. J. Fluid Mech. 615, 121-138.

BALAKUMAR, B. J. \& ADRIAN, R. J. 2007 Large- and very-large-scale motions in channel and boundary-layer flows. Phil. Trans. R. Soc. Lond. A 365, 665-681.

BAmieh, B. \& DAhleh, M. 2001 Energy amplification in channel flows with stochastic excitation. Phys. Fluids 13 (11), 3258-3269.

Bourguignon, J.-L., Sharma, A. S., Tropp, J. A. \& McKeon, B. J. 2013 Compact representation of wall-bounded turbulence using compressive sampling (submitted).

Butler, K. M. \& FARrell, B. F. 1992 Three-dimensional optimal perturbations in viscous shear flow. Phys. Fluids A 4, 1637-1650.

Butler, K. M. \& FARRell, B. F. 1993 Optimal perturbations and streak spacing in wall-bounded turbulent shear flow. Phys. Fluids A 5 (3), 774-777.

CESS, R. D. 1958 A survey of the literature on heat transfer in turbulent tube flow. Westinghouse Research, Rep. 8-0529-R24.

Chung, D. \& MCKeON, B. J. 2010 Large-eddy simulation investigation of large-scale structures in a long channel flow. J. Fluid Mech. 661, 341-364.

Coles, D. E. 1956 The law of the wake in the turbulent boundary layer. J. Fluid Mech. 1, 191-226.

CVX RESEARCH, INC., 2012 CVX: Matlab software for disciplined convex programming, version 2.0 beta. http://cvxr.com/cvx.

DE GRAAFF, D. B. \& EATON, J. K. 2000 Reynolds-number scaling of the flat-plate turbulent boundary layer. J. Fluid Mech. 422, 319-346.

FARRELl, B. F. \& IOANNOU, P. J. $1993 a$ Optimal excitation of three-dimensional perturbations in viscous constant shear flow. Phys. Fluids A 5 (6), 1390-1400.

FARrell, B. F. \& IOANnOU, P. J. $1993 b$ Stochastic forcing of the linearized Navier-Stokes equations. Phys. Fluids A 5 (11), 2600-2609.

FARRELl, B. F. \& IOANNOU, P. J. 1998 Perturbation structure and spectra in turbulent channel flow. Theor. Comput. Fluid Dyn. 11, 237-250.

Fernholz, H. H., Krause, E., Nockemann, M. \& Schober, M. 1995 Comparative measurements in the canonical boundary layer at $R_{\theta} \leqslant 6 \times 10^{4}$ on the wall of the German-Dutch windtunnel. Phys. Fluids 7, 1275-1281.

Flores, O. \& JiMÉneZ, J. 2010 Hierarchy of minimal flow units in the logarithmic layer. Phys. Fluids 22, 071704.

Gad-El-Hak, M. \& BAndyopadhyay, P. R. 1994 Reynolds number effects in wall-bounded turbulent flows. Appl. Mech. Rev. 47 (8), 307-365.

GRANT, M. \& BOYD, S. 2008 Graph implementations for nonsmooth convex programs. In Recent Advances in Learning and Control (ed. V. Blondel, S. Boyd \& H. Kimura), pp. 95-110. Springer, http://stanford.edu/ boyd/graph_dcp.html.

Guala, M., Hommema, S. E. \& Adrian, R. J. 2006 Large-scale and very-large-scale motions in turbulent pipe flow. J. Fluid Mech. 554, 521-542.

Gustavsson, L. H. 1991 Energy growth of three-dimensional disturbances in plane Poiseuille flow. J. Fluid Mech. 224, 241-260. 
Halko, N., Martinsson, P. G. \& Tropp, J. A. 2011 Finding structure with randomness: probabilistic algorithms for constructing approximate matrix decompositions. SIAM Rev. $\mathbf{5 3}$ (2), 217-288.

Hamilton, J. M., Kim, J. \& Waleffe, F. 1995 Regeneration mechanisms of near-wall turbulence structures. J. Fluid Mech. 287, 317-348.

HEAD, M. R. \& BANDYOPADHYAY, P. 1981 New aspects of turbulent boundary-layer structure. J. Fluid Mech. 107, 297-338.

HoYAS, S. \& JimÉNEZ, J. 2006 Scaling of the velocity fluctuations in turbulent channels up to $R e_{\tau}=2003$. Phys. Fluids 18 (1), 011702.

Hultmark, M., Vallikivi, M., Bailey, S. C. C. \& Smits, A. J. 2012 Turbulent pipe flow at extreme Reynolds numbers. Phys. Rev. Lett. 108 (9), 94501.

Hutchins, N. \& MARUsic, I. 2007a Evidence of very long meandering features in the logarithmic region of turbulent boundary layers. J. Fluid Mech. 579, 1-28.

Hutchins, N. \& MARUsic, I. $2007 b$ Large-scale influences in near-wall turbulence. Phil. Trans. R. Soc. Lond. A 365, 647-664.

Hutchins, N., Monty, J. P., Ganapathisubramani, B., Ng, H. C. H. \& Marusic, I. 2011 Three-dimensional conditional structure of a high-Reynolds number turbulent boundary layer. J. Fluid Mech. 673, 255-285.

Hutchins, N., Nickels, T. B., Marusic, I. \& Chong, M. S. 2009 Hot-wire spatial resolution issues in wall-bounded turbulence. J. Fluid Mech. 635, 103-136.

HWANG, Y. \& Cossu, C. 2010 Linear non-normal energy amplification of harmonic and stochastic forcing in the turbulent channel flow. J. Fluid Mech. 664, 51-73.

Hwang, Y. \& Cossu, C. 2011 Self-sustained processes in the logarithmic layer of turbulent channel flows. Phys. Fluids 23, 061702.

Inoue, M., Mathis, R., Marusic, I. \& Pullin, D. I. 2012 Inner-layer intensities for the flat-plate turbulent boundary layer combining a predictive wall-model with large-eddy simulations. Phys. Fluids 24, 075102.

JimÉnEZ, J. 2012 Cascades in wall-bounded turbulence. Annu. Rev. Fluid Mech. 44, 27-45.

JimÉNEZ, J. \& HoYAS, S. 2008 Turbulent fluctuations above the buffer layer of wall-bounded flows. J. Fluid Mech. 611, 215-236.

Jovanović, M. R. \& BAmieH, B. 2005 Componentwise energy amplification in channel flows. J. Fluid Mech. 534, 145-183.

KeRSWELL, R. R. 2005 Recent progress in understanding the transition to turbulence in a pipe. Nonlinearity 18, R17-R44.

KIM, K. C. \& AdRIAN, R. J. 1999 Very large-scale motion in the outer layer. Phys. Fluids 11, $417-422$.

KIM, J. \& HusSAIN, F. 1993 Propagation velocity of perturbations in turbulent channel flow. Phys. Fluids A 5, 695-706.

KIM, J. \& LiM, J. 2000 A linear process in wall-bounded turbulent shear flows. Phys. Fluids 12 (8), $1885-1888$.

KLEWICKI, J. C. 2010 Reynolds number dependence, scaling, and dynamics of turbulent boundary layers. Trans. ASME: J. Fluids Engng 132 (9), 094001.

Kline, S. J., Reynolds, W. C., Schraub, F. A. \& Runstadler, P. W. 1967 The structure of turbulent boundary layers. J. Fluid Mech. 30, 741-773.

Klingmann, B. G. B. 1992 On transition due to three-dimensional disturbances in plane Poiseuille flow. J. Fluid Mech. 240, 167-195.

Kunkel, G. J. \& MARUSIC, I. 2006 Study of the near-wall-turbulent region of the high-Reynoldsnumber boundary layer using an atmospheric flow. J. Fluid Mech. 548, 375-402.

LANDAHL, M. T. 1975 Wave breakdown and turbulence. SIAM J. Appl. Maths 28, 735-756.

LeHew, J., Guala, M. \& McKeon, B. J. 2011 A study of the three-dimensional spectral energy distribution in a zero pressure gradient turbulent boundary layer. Exp. Fluids 51, 997-1012.

Long, R. R. \& Chen, T. C. 1981 Experimental evidence for the existence of the mesolayer in turbulent systems. J. Fluid Mech. 105, 19-59.

Malkus, W. V. R. 1956 Outline of a theory of turbulent shear flow. J. Fluid Mech. 1 (5), 521-539. 
Marusic, I. \& KUnKel, G. J. 2003 Streamwise turbulence intensity formulation for flat-plate boundary layers. Phys. Fluids 15 (8), 2461-2464.

Marusic, I., Mathis, R. \& Hutchins, N. 2010a High Reynolds number effects in wall turbulence. Intl J. Heat Fluid Flow 31, 418-428.

Marusic, I., Mathis, R. \& Hutchins, N. $2010 b$ Predictive model for wall-bounded turbulent flow. Science 329 (5988), 193-196.

Marusic, I., McKeon, B. J., Monkewitz, P. A., Nagib, H. M., Smits, A. J. \& SREENIVASAN, K. R. 2010c Wall-bounded turbulent flows at high Reynolds numbers: recent advances and key issues. Phys. Fluids 22, 065103.

Marusic, I., Monty, J. P., Hultmark, M. \& Smits, A. J. 2013 On the logarithmic region in wall turbulence. J. Fluid Mech. 716, R3-1-716-R3-11.

Mathis, R., Hutchins, N. \& MARUsic, I. 2009a Large-scale amplitude modulation of the small-scale structures in turbulent boundary layers. J. Fluid Mech. 628, 311-337.

Mathis, R., Monty, J. P., Hutchins, N. \& Marusic, I. 2009b Comparison of large-scale amplitude modulation in turbulent boundary layers, pipes, and channel flows. Phys. Fluids 21, 111703.

Matsubara, M. \& Alfredsson, P. H. 2001 Disturbance growth in boundary layers subjected to free stream turbulence. J. Fluid Mech. 430, 149-168.

McKeon, B. J. \& Sharma, A. S. 2010 A critical-layer framework for turbulent pipe flow. J. Fluid Mech. 658, 336-382.

McKeon, B. J., Sharma, A. S. \& Jacobi, I. 2013 Experimental manipulation of wall turbulence: a systems approach. Phys. Fluids 25, 031301.

Meseguer, A. \& Trefethen, L. N. 2003 Linearized pipe flow to Reynolds number $10^{7}$. J. Comput. Phys. 186, 178-197.

Metzger, M. M. \& KLEwicki, J. C. 2001 A comparative study of near-wall turbulence in high and low Reynolds number boundary layers. Phys. Fluids 13, 692-701.

Mizuno, Y. \& JimÉneZ, J. 2013 Wall turbulence without walls. J. Fluid Mech. 723, 429-455.

MoArref, R. \& Jovanović, M. R. 2012 Model-based design of transverse wall oscillations for turbulent drag reduction. J. Fluid Mech. 707, 205-240.

Monty, J. P. 2005 Developments in smooth wall turbulent duct flows. PhD thesis, University of Melbourne.

Monty, J. P. \& ChOng, M. S. 2009 Turbulent channel flow: comparison of streamwise velocity data from experiments and direct numerical simulation. J. Fluid Mech. 633, 461-474.

Monty, J. P., Hutchins, N., NG, H. C. H., Marusic, I. \& Chong, M. S. 2009 A comparison of turbulent pipe, channel and boundary layer flows. J. Fluid Mech. 632, 431-442.

Monty, J. P., Stewart, J. A., Williams, R. C. \& Chong, M. S. 2007 Large-scale features in turbulent pipe and channel flows. J. Fluid Mech. 589, 147-156.

Morrison, J. F., McKeon, B. J., JiAng, W. \& Smits, A. J. 2004 Scaling of the streamwise velocity component in turbulent pipe flow. J. Fluid Mech. 508, 99-131.

Nagib, H. M. \& Chauhan, K. A. 2008 Variations of von Kármán coefficient in canonical flows. Phys. Fluids 20, 101518.

Panton, R. L. 2001 Overview of the self-sustaining mechanisms of wall turbulence. Prog. Aerosp. Sci. 37 (4), 341-383.

Perry, A. E. \& Chong, M. S. 1982 On the mechanism of wall turbulence. J. Fluid Mech. 119, $106-121$.

Pujals, G., García-Villalba, M., Cossu, C. \& Depardon, S. 2009 A note on optimal transient growth in turbulent channel flows. Phys. Fluids 21 (1), 015109.

Reddy, S. C. \& Henningson, D. S. 1993 Energy growth in viscous channel flows. J. Fluid Mech. 252, 209-238.

Reynolds, W. C. \& Hussain, A. K. M. F. 1972 The mechanics of an organized wave in turbulent shear flow. Part 3. Theoretical models and comparisons with experiments. J. Fluid Mech. 54 (2), 263-288.

Reynolds, W. C. \& Tiederman, W. G. 1967 Stability of turbulent channel flow with application to Malkus's theory. J. Fluid Mech. 27 (2), 253-272.

Robinson, S. K. 1991 Coherent motions in the turbulent boundary layer. Annu. Rev. Fluid Mech. 23, 601-639. 
SCHMid, P. J. 2007 Nonmodal stability theory. Annu. Rev. Fluid Mech. 39, 129-162.

SCHMid, P. J. \& HenNingson, D. D. 1994 Optimal energy density growth in Hagen-Poiseuille flow. J. Fluid Mech. 277, 197-225.

Schmid, P. J. \& Henningson, D. S. 2001 Stability and Transition in Shear Flows. Springer.

Schoppa, W. \& Hussain, F. 2002 Coherent structure generation in near-wall turbulence. J. Fluid Mech. 453, 57-108.

Schultz, M. P. \& FlaCK, K. A. 2013 Reynolds-number scaling of turbulent channel flow. Phys. Fluids 25, 025104.

Sharma, A. S. \& MCKeon, B. J. 2013 On coherent structure in wall turbulence. J. Fluid Mech. 728, 196-238.

Smith, C. R. \& Metzler, S. P. 1983 The characteristics of low-speed streaks in the near-wall region of a turbulent boundary layer. J. Fluid Mech. 129, 27-54.

Smits, A. J., McKeon, B. J. \& Marusic, I. 2011 High-Reynolds number wall turbulence. Annu. Rev. Fluid Mech. 43, 353-375.

SReEnivasan, K. R. \& SAHAY, A. 1997 The persistence of viscous effects in the overlap region, and the mean velocity in turbulent pipe and channel flows. In Self-Sustaining Mechanisms of Wall Turbulence (ed. R. Panton), pp. 253-272. Comp. Mech. Publ..

TAYlor, G. 1938 The spectrum of turbulence. Proc. R. Soc. Lond. A 164 (919), 476-490.

TOMKINS, C. D. \& ADRIAN, R. J. 2003 Spanwise structure and scale growth in turbulent boundary layers. J. Fluid Mech. 490, 37-74.

Townsend, A. 1976 The Structure of Turbulent Shear Flow. Cambridge University Press.

Trefethen, L. N., Trefethen, A. E., Reddy, S. C. \& Driscoll, T. A. 1993 Hydrodynamic stability without eigenvalues. Science 261, 578-584.

WalefFe, F. 1997 On a self-sustaining process in shear flows. Phys. Fluids 9 (4), 883-900.

WALEFFE, F. 2003 Homotopy of exact coherent structures in plane shear flows. Phys. Fluids 15, $1517-1534$.

WEDIN, H. \& KERSWELL, R. R. 2004 Exact coherent structures in pipe flow: travelling wave solutions. J. Fluid Mech. 508, 333-371.

Wei, T., Fife, P., Klewicki, J. \& McMurtry, P. 2005 Properties of the mean momentum balance in turbulent boundary layer, pipe and channel flows. J. Fluid Mech. 522, 303-327.

Weideman, J. A. C. \& Reddy, S. C. 2000 A MATLAB differentiation matrix suite. ACM Trans. Math. Softw. 26 (4), 465-519. 